\title{
A PRIORI ERROR ESTIMATES FOR NUMERICAL METHODS FOR SCALAR CONSERVATION LAWS. PART I: THE GENERAL APPROACH
}

\author{
BERNARDO COCKBURN AND PIERRE-ALAIN GREMAUD
}

\begin{abstract}
In this paper, we construct a general theory of a priori error estimates for scalar conservation laws by suitably modifying the original Kuznetsov approximation theory. As a first application of this general technique, we show that error estimates for conservation laws can be obtained without having to use explicitly any regularity properties of the approximate solution. Thus, we obtain optimal error estimates for the Engquist-Osher scheme without using the fact (i) that the solution is uniformly bounded, (ii) that the scheme is total variation diminishing, and (iii) that the discrete semigroup associated with the scheme has the $\mathrm{L}^{1}$-contraction property, which guarantees an upper bound for the modulus of continuity in time of the approximate solution.
\end{abstract}

\section{INTRODUCTION}

This is the first of a series of papers in which we develop a theory of a priori error estimates, that is, estimates given solely in terms of the exact solution, for numerical methods for the scalar conservation law [14]

$$
\begin{array}{ll}
v_{t}+\nabla \cdot f(v)=0 & \text { in }(0, T) \times \mathbb{R}^{d}, \\
v(0)=v_{0} & \text { on } \mathbb{R}^{d} .
\end{array}
$$

Our main long-term goal is to obtain a theory of a priori error estimates which

(i) can be applied to a large class of numerical schemes,

(ii) does not require regularity properties of the approximate solution,

(iii) takes into account the properties of the triangulation,

(iv) takes into account the smoothness of the exact solution.

In this paper, we show how to obtain such a theory by a slight modification of the original Kuznetsov approximation theory for conservation laws [15]. We then give an application with the purpose of stressing the point (ii) above. Thus, we show how to obtain optimal error estimates for the Engquist-Osher scheme without explicitly using any regularity property of the approximate solution. Unlike previous work, we do not make use of the fact that the approximate solution is uniformly bounded, nor that the scheme is total variation diminishing, nor do we use the

Received by the editor August 22, 1994 and, in revised form, February 22, 1995.

1991 Mathematics Subject Classification. Primary 65M60, 65N30, 35L65.

Key words and phrases. A priori error estimates, monotone schemes, conservation laws.

First author partially supported by the National Science Foundation (Grant DMS-9407952) and by the University of Minnesota Supercomputer Institute.

Second author partially supported by the University of Minnesota Supercomputer Institute. 
$\mathrm{L}^{1}$-contraction property of the discrete semigroup, which leads to an estimate of the modulus of continuity in time of the approximate solution. Instead, we use the regularity properties of the entropy solution.

A theory of a priori error estimates with the above properties does not exist for scalar conservation laws. To shed light into this unfortunate situation, we briefly describe the historical development of the theory of error estimates for conservation laws. As a tool for illustration, let us consider the problem of obtaining an upper bound of the distance between the entropy solution $v$ of (1.1) and the bounded solution $u$ of the following parabolic initial value problem:

$$
\begin{array}{ll}
u_{t}+\nabla \cdot f(u)-\nabla \cdot(\nu(u) \nabla u)=0 & \text { in }(0, T) \times \mathbb{R}^{d}, \\
u(0)=v_{0} & \text { on } \mathbb{R}^{d},
\end{array}
$$

where $\nu$ is a positive nonlinear function. It is very natural to consider this problem since it is well known that the entropy solution $v$ can be obtained as the limit of solutions $u$ of the above initial parabolic problem as the viscosity $\nu$ goes to zero; see [14]. Moreover, several numerical schemes of interest have equation (1.2a) as their so-called model equation. This means that it is reasonable to expect that both $u$ and the approximate solution of the corresponding numerical scheme would behave in a similar way; see, for example, [12] and [11]. The method of obtaining error estimates for $u$ would then constitute a model for obtaining error estimates for the corresponding numerical scheme.

Kuznetsov [15] was the first to prove error estimates for numerical schemes for (1.1). Inspired by the work of Kružkov [14], he established a key approximation inequality [15, Lemma 2] with which he obtained the following estimate [15, Theorem 3]:

$$
\|u(T)-v(T)\|_{L^{1}\left(\mathbb{R}^{d}\right)} \leq \sqrt{8}\left|v_{0}\right|_{T V\left(\mathbb{R}^{d}\right)}^{1 / 2}\left\{\int_{0}^{T} \int_{\mathbb{R}^{d}} \nu(u)|\nabla u| d x d t\right\}^{1 / 2}
$$

Kuznetsov then applied the same approach to obtain error estimates for monotone schemes in uniform Cartesian grids. Since these schemes have a model equation of the form (1.2a), where the viscosity $\nu$ is proportional to the grid size $\Delta x$, it was natural to use the approach used for $u$ as the model approach for this, more complicated, case. As the model inequality above indicates, Kuznetsov was bound to obtain both an estimate of the total variation and of the uniform norm of the approximate solutions determined by the monotone schemes he considered. He proved that the total variation and the uniform norm are uniformly bounded with respect to $\Delta x$ and concluded that monotone schemes in uniform Cartesian grids converge as $(\Delta x)^{1 / 2}$ to the entropy solution in the $\mathrm{L}^{\infty}\left(\mathrm{L}^{1}\right)$-norm. This pioneering work strongly influenced the subsequent studies of error estimates for numerical schemes for (1.1). Thus, the works of Sanders [22], Lucier [17,18,19], and Cockburn $[1,2,3]$ used the approach used by Kuznetsov and had to obtain regularity properties of the approximate solution to obtain error estimates. In particular, they all used the fact that the total variation of the approximate solution was uniformly bounded.

It soon became apparent that progress along the above approach was going to be very hard to achieve, since even the simplest schemes, the monotone schemes, 
could not be proven to generate approximate solutions with uniformly bounded total variation when defined in general triangulations. To obtain error estimates thus became an extremely difficult task, and the main focus of research shifted to the search of weaker smoothness properties with which convergence, not error estimates, could be proven. Because of this, DiPerna's theory of convergence of measure-valued functions [9] became the main tool for the study of the convergence of numerical schemes for the conservation (1.1). Szepessy [23, 24] was the first to recognize this fact and proved convergence to the entropy solution of (1.1) of the approximate solution given by the Streamline Diffusion method with shockcapturing terms. Later, Coquel and LeFloch [8] applied DiPerna's theory to finite difference schemes in Cartesian grids, and Cockburn, Coquel and LeFloch [4] to finite volume methods defined in general triangulations.

A third stage in the development of the theory of error estimates for scalar conservation laws started when Cockburn, LeFloch and Coquel [5] realized that the measure-valued theory of DiPerna could be totally bypassed by using a suitable modification of the Kuznetsov approximation result; after all, both DiPerna and Kuznetsov were inspired by the same source, namely, the paper by Kružkov [14]. Error estimates were thus obtained with (essentially) the same hypotheses used to prove convergence with the measure-valued solutions approach. More precisely, in [5], it was proven that monotone schemes defined in general triangulations, and some high-order accurate schemes built upon them, converge to the entropy solution finite volume methods with a rate of no less than $(\Delta x)^{1 / 4}$ in the $\mathrm{L}^{\infty}\left(\mathrm{L}^{1}\right)$-norm. Also, Cockburn and Gremaud [7] proved that the shock-capturing Discontinuous Galerkin method converges with a rate of no less than $(\Delta x)^{1 / 4}$ in the $\mathrm{L}^{\infty}\left(\mathrm{L}^{1}\right)$-norm and that the shock-capturing Streamline Diffusion method converges with a rate of no less than $(\Delta x)^{1 / 8}$ in the $\mathrm{L}^{\infty}\left(\mathrm{L}^{1}\right)$-norm. Two new ideas made these results possible. The first idea was a new way of estimating the total variation of the approximate solution which, in terms of our parabolic regularization $u$, reduces to the standard $\mathrm{L}^{2}$-stability result,

$$
\|u(T)\|_{L^{2}\left(\mathbb{R}^{d}\right)}^{2}+2 \int_{0}^{T} \int_{\mathbb{R}^{d}} \nu(u)|\nabla u|^{2} d x d t \leq\left\|v_{0}\right\|_{L^{2}\left(\mathbb{R}^{d}\right)}^{2},
$$

easily obtained by multiplying equation (1.2a) by $u$ and integrating over $(0, T) \times \mathbb{R}^{d}$; see $[4,13,7$, and 26]. Combining this stability result with the estimate (1.3), we obtain

$$
\|u(T)-v(T)\|_{L^{1}\left(\mathbb{R}^{d}\right)} \leq \frac{\sqrt{8}}{2^{1 / 4}}\left|v_{0}\right|_{T V\left(\mathbb{R}^{d}\right)}^{1 / 2}\left\|v_{0}\right\|_{L^{2}\left(\mathbb{R}^{d}\right)}^{1 / 2}\left\{\int_{0}^{T} \int_{\mathbb{R}^{d}} \nu(u) d x d t\right\}^{1 / 4} .
$$

This explains, in a nutshell, the rate of convergence of $(\Delta x)^{1 / 4}$ for monotone schemes obtained in [5] and in [26].

The second idea requires more elaboration. For the sake of clarity, we did not mention that Kuznetsov approximation inequality can be used, in the general case, only if an estimate of the modulus of continuity in time of the approximate solution is available. For monotone schemes, this estimate follows easily from an estimate of the total variation and from the $\mathrm{L}^{1}$-contraction property of these schemes. However, this property does not hold for other types of schemes. The second idea consists in modifying the original Kuznetsov approximation inequality so that no estimate of 
the modulus of continuity in time is needed anymore. This was done in [5] for finite volume methods and in [7] for the shock-capturing Discontinuous Galerkin and the shock-capturing Streamline Diffusion methods. An application of this idea to the model case under consideration gives the following error estimate:

$$
\|u(T)-v(T)\|_{L^{1}\left(\mathbb{R}^{d}\right)} \leq 4 \sqrt{8}\left|v_{0}\right|_{T V\left(\mathbb{R}^{d}\right)}^{1 / 2}\left\{\int_{0}^{T} \int_{\mathbb{R}^{d}} \nu(u)|\nabla u| d x d t\right\}^{1 / 2},
$$

which shows that the (very small!) price we have to pay for not having to estimate the modulus of continuity in time of the approximating function $u$ is a constant 4 times bigger multiplying the upper bound of the error (compare with the estimate (1.3)!). If now we apply the estimate of the total variation (1.4), we get

$$
\|u(T)-v(T)\|_{L^{1}\left(\mathbb{R}^{d}\right)} \leq \frac{4 \sqrt{8}}{2^{1 / 4}}\left|v_{0}\right|_{T V\left(\mathbb{R}^{d}\right)}^{1 / 2}\left\|v_{0}\right\|_{L^{2}\left(\mathbb{R}^{d}\right)}^{1 / 2}\left\{\int_{0}^{T} \int_{\mathbb{R}^{d}} \nu(u) d x d t\right\}^{1 / 4} .
$$

This is a rough explanation of the rate of convergence of $(\Delta x)^{1 / 4}$ obtained in [5], for a class of high-order accurate antidiffusion schemes, and in [7], for the shockcapturing Discontinuous Galerkin method.

To the knowledge of the authors, this is the current status of the theory of error estimates for conservation laws. It is indeed not very encouraging to realize that after all the effort reported above, the orders of convergence, namely, $1 / 2,1 / 4,1 / 8$, seem to be converging to the wrong limit! However, we claim that a slight change of point of view in the original Kuznetsov approach can dramatically improve this situation. As noted by Cockburn and Gremaud [7], Kuznetsov's approach is essentially an approach leading to a posteriori error estimates, since the upper bound for the error is given in terms of the approximate solution; see (1.3)! As a consequence, it is not surprising to be forced to prove regularity properties of the approximate solution in order to obtain a rate of convergence. Thus, Kuznetsov's approach should be used for obtaining a posteriori with which adaptivity strategies could be defined; see the work by Lucier in $[16,19]$ and the recent work by Cockburn and Gau [6]. In this paper, we show that this new point of view induces, in a very natural way, a slight change in Kuznetsov's approach with which we construct a general procedure for obtaining a priori error estimates, that is, for obtaining upper bounds for the error that depend solely on the exact solution.

The paper is organized as follows. In $\S 2$, we start by rewriting the original Kuznetsov approach in our own notation. This allows us to compare Kuznetsov's approach with our new approach, which is described in $\S 3$. The remaining of the paper is devoted to the application of this new approach to two relatively simple, but relevant, cases with the main purposes (i) of clearly illustrating the new procedure, and (ii) of showing that it is possible to obtain optimal error estimates for conservation laws without having to establish any regularity properties of the approximate solution. Thus, we obtain optimal error estimates for (i) the parabolic solution of (1.2), Theorem 4.1, and for (ii) the approximate solution given by the well-known Engquist-Osher scheme in uniform grids, Theorem 6.1; we consider only the one-dimensional case $d=1$ for the sake of simplicity. The proof of Theorem 4.1 is given in $\S 5$. It provides a simple 'map' for the more involved proof of Theorem 6.1 , given in $\S 7$. We end in $\S 8$ with some concluding remarks concerning extensions 
of the above results to the multidimensional case, and a brief description of future work.

The a priori estimates presented in Theorems 4.1 and 6.1 are the first of its type for nonlinear scalar conservation laws. For the steady-state Hamilton-Jacobi equations, Perthame and Sanders [21, Theorem 2] obtained error estimates which in some special cases can be considered a priori in the sense used in this paper; in the general case, their upper bound for the error does depend on the $\mathrm{L}^{\infty}$-norm of the approximate solution.

\section{KUZNETSOV'S ORIGINAL APPROACH}

Since our approach to obtaining error estimates for the conservation law (1.1) is a modification of Kuznetsov's original approach, in this section we rewrite Kuznetsov's approach step by step in our notation. This will help us to motivate the steps of our approach and to render clear the similarities and differences of the two approaches. To fix ideas, in what follows, $u=u\left(t^{\prime}, x^{\prime}\right)$ stands for the bounded solution of the parabolic problem (1.2) and the function $v=v(t, x)$ stands for the entropy solution of the conservation law (1.1).

a. Choosing the entropy dissipation form $E\left(u, v ; t^{N}\right)$. Kuznetsov picked the so-called Kruškov form

$$
E(u, v ; \tau)=\int_{0}^{\tau} \int_{\mathbb{R}^{d}} \Theta_{\tau}(u, v(t, x) ; t, x) d x d t,
$$

where

$$
\begin{aligned}
\Theta_{\tau}(u, c ; t, x)= & -\int_{0}^{\tau} \int_{\mathbb{R}^{d}} U\left(u\left(t^{\prime}, x^{\prime}\right)-c\right) \partial_{t^{\prime}} \varphi d x^{\prime} d t^{\prime} \\
& -\int_{0}^{\tau} \int_{\mathbb{R}^{d}} F\left(u\left(t^{\prime}, x^{\prime}\right), c\right) \cdot \nabla_{x^{\prime}} \varphi d x^{\prime} d t^{\prime} \\
& +\int_{\mathbb{R}^{d}} U\left(u\left(\tau, x^{\prime}\right)-c\right) \varphi\left(t, x, \tau, x^{\prime}\right) d x^{\prime} \\
& -\int_{\mathbb{R}^{d}} U\left(u\left(0, x^{\prime}\right)-c\right) \varphi\left(t, x, 0, x^{\prime}\right) d x^{\prime}
\end{aligned}
$$

and the entropy $U$ and its flux $F$ (that is, $\partial_{w} F(w, c)=f^{\prime}(w) U^{\prime}(w-c)$ ) are given by

$$
\begin{aligned}
& U(w-c)=|w-c|, \\
& F(w, c)=(f(w)-f(c)) \operatorname{sign}(w-c) .
\end{aligned}
$$

The function $\varphi=\varphi\left(t, x, t^{\prime}, x^{\prime}\right)$ is taken as follows:

$$
\varphi=w_{\epsilon_{t}}\left(t-t^{\prime}\right) \prod_{i=1}^{d} w_{\epsilon_{x}}\left(x_{i}-x_{i}^{\prime}\right), \quad(x, t),\left(x^{\prime}, t^{\prime}\right) \in \mathbb{R}^{d} \times \mathbb{R}^{+},
$$

where $\epsilon_{t}$ and $\epsilon_{x}$ are two arbitrary positive numbers and

$$
w_{\lambda}(s)=\frac{1}{\lambda} w\left(\frac{s}{\lambda}\right),
$$


for any $s \in \mathbb{R}, \lambda=\epsilon_{t}, \epsilon_{x}$. The function $w$ is a smooth function satisfying the following properties:

$$
\begin{aligned}
& w(t) \geq 0 \text { for } t>0 \\
& w(t)=w(-t) \text { for } t>0, \\
& \text { the support of } w \text { is }[-1,1] \\
& \int_{0}^{1} w(r) d r=1 / 2
\end{aligned}
$$

Note that $\varphi\left(t, x, t^{\prime}, x^{\prime}\right)$ is a smooth approximation of $\delta\left(t-t^{\prime}\right) \cdot \delta\left(x-x^{\prime}\right)$.

There are two main motivations for this choice of $E(u, v ; \tau)$. The first is that if $u$ is the entropy solution of (1.1) with initial data $v_{0}=u_{0}$, then $u$ satisfies the so-called entropy inequality, or, equivalently, $\Theta_{\tau}^{\epsilon_{t}, \epsilon_{x}}(u, c ; t, x) \leq 0$ for $c \in \mathbb{R}$, and $(t, x) \in(0, T) \times \mathbb{R}^{d}$. This implies that $E(u, v ; \tau) \leq 0$. Thus, the form $E(u, v ; \tau)$ measures how close from being an entropy solution the approximate solution $u$ is. The other reason will become apparent in the next step.

b. The dual form $E^{\star}(u, v ; \tau)$ and the error term $T_{\operatorname{err}}(u, v ; \tau)$. In this step, a dual form $E^{\star}(u, v ; \tau)$ and an error term $T_{\operatorname{err}}(u, v ; \tau)$ are found such that

$$
\begin{aligned}
E(u, v ; \tau) & =-E^{\star}(u, v ; \tau)+T_{\operatorname{err}}(u, v ; \tau) \\
& =-E(v, u ; \tau)+T_{\operatorname{err}}(u, v ; \tau),
\end{aligned}
$$

by solely taking into account the following equalities:

$$
\begin{aligned}
& \partial_{t} \varphi=-\partial_{t^{\prime}} \varphi \\
& \nabla_{x} \varphi=-\nabla_{x^{\prime}} \varphi \\
& U(w-c)=U(c-w), \\
& F(w, c)=F(c, w) .
\end{aligned}
$$

Since $v$ is the entropy solution, we have that $E^{\star}(u, v ; \tau)=E(v, u ; \tau) \leq 0$ (this is the other main reason for choosing the form $E(u, v ; \tau)$ as in the first step), and we obtain

$$
\begin{aligned}
T_{\mathrm{err}}(u, v ; \tau) & =E(u, v ; \tau)+E(v, u ; \tau) \\
& \leq E(u, v ; \tau)
\end{aligned}
$$

where the error term $T_{\operatorname{err}}(u, v ; \tau)$ contains the information of the distance between the functions $u$ and $v$. In fact, as the parameters $\epsilon_{t}$ and $\epsilon_{x}$ defining $\varphi$ go to zero, we expect the term $T_{\text {err }}(u, v ; \tau)$ to converge to

$$
e(\tau)-e(0)
$$

where the error $e(t)$ is given by

$$
e(t)=\int_{\mathbb{R}} U(u(t, x)-v(t, x)) d x .
$$

This is why the term $T_{\text {err }}(u, v ; \tau)$ is called the "error term". 
c. Finding a lower bound of the error term $T_{\operatorname{err}}(u, v ; \tau)$. Setting

$$
W(t)=\int_{0}^{t} w_{\epsilon_{t}}(s) d s
$$

we can rewrite Kuznetsov's lower bound for the error term as follows:

$$
\begin{aligned}
T_{\mathrm{err}}(u, v ; \tau) \geq & 2 W(\tau) e(\tau)-2 W(\tau) e(0) \\
& -2 W(\tau)|v|_{L^{\infty}\left(0, \tau ; T V\left(\mathbb{R}^{d}\right)\right)}\left\{\epsilon_{x}+\left\|f^{\prime}(v)\right\| \epsilon_{t}\right\} \\
& -2 W(\tau)\left\{\epsilon_{x}\left\|\nabla_{x^{\prime}} u\right\|_{L^{\infty}\left(0, \tau ; L^{1}\left(\mathbb{R}^{d}\right)\right)}+\epsilon_{t}\left\|u_{t^{\prime}}\right\|_{L^{\infty}\left(0, \tau ; L^{1}\left(\mathbb{R}^{d}\right)\right)}\right\},
\end{aligned}
$$

where $\left\|f^{\prime}(v)\right\|=\sup _{\substack{t \in(0, \tau) \\ x \in \mathbb{R}^{d}}}\left|f^{\prime}(v(t, x))\right|_{L^{\infty}}$. Kuznetsov obtained this inequality for $\tau \geq \epsilon_{t}$ only, a case in which we have $2 W(\tau)=1$.

d. Finding an upper bound for the error $e(T)$. Putting together all the results of the previous steps, we obtain the following inequality:

$$
\begin{aligned}
e(T) \leq & e(0)+\left|v_{0}\right|_{T V\left(\mathbb{R}^{d}\right)}\left\{\epsilon_{x}+\left\|f^{\prime}(v)\right\| \epsilon_{t}\right\}+E(u, v ; T) / 2 W(T) \\
& +\left\{\epsilon_{x}\left\|\nabla_{x^{\prime}} u\right\|_{L^{\infty}\left(0, \tau ; L^{1}\left(\mathbb{R}^{d}\right)\right)}+\epsilon_{t}\left\|u_{t^{\prime}}\right\|_{L^{\infty}\left(0, \tau ; L^{1}\left(\mathbb{R}^{d}\right)\right)}\right\} .
\end{aligned}
$$

e. Estimating $E(u, v ; T) / W(T)$ and getting regularity properties of $u$. At this point, to get an error estimate we only have to estimate the Kruškov form $E(u, v ; T) / W(T)$, the total variation of $u$ and its modulus of continuity in time. To estimate the Kruškov form, the form is first split into the sum of two terms,

$$
E(u, v ; T)=-E_{\mathrm{diss}}(u, v ; T)+E_{\mathrm{reg}}(u, v ; T),
$$

which, in this case, are estimated as follows:

$$
\begin{aligned}
E_{\mathrm{diss}}(u, v ; T) & =\int_{0}^{\tau} \int_{\mathbb{R}^{d}} \int_{0}^{\tau} \int_{\mathbb{R}^{d}} U^{\prime \prime}(u-v) \nu(u)|\nabla u|^{2} \varphi d x^{\prime} d t^{\prime} d x d t \\
& \geq 0, \\
E_{\mathrm{reg}}(u, v ; T) / W(\tau) & =\int_{0}^{\tau} \int_{\mathbb{R}^{d}} \int_{0}^{\tau} \int_{\mathbb{R}^{d}} \nabla \cdot(\nu(u) \nabla U(u-v)) \varphi d x^{\prime} d t^{\prime} d x d t / W(\tau) \\
& \leq \frac{2}{\epsilon_{x}}\left\{\int_{0}^{\tau} \int_{\mathbb{R}^{d}} \nu(u)|\nabla u| d x^{\prime} d t^{\prime}\right\} .
\end{aligned}
$$

We also have

$$
\begin{aligned}
& \|\nabla u\|_{L^{\infty}\left(0, \tau ; L^{1}\left(\mathbb{R}^{d}\right)\right)} \leq\left|u_{0}\right|_{T V\left(\mathbb{R}^{d}\right)}, \\
& \left\|u_{t^{\prime}}\right\|_{L^{\infty}\left(0, \tau ; L^{1}\left(\mathbb{R}^{d}\right)\right)} \leq\left\|f^{\prime}(u)\right\|\left|u_{0}\right|_{T V\left(\mathbb{R}^{d}\right)} .
\end{aligned}
$$

f. Getting the error estimate. The error estimate follows now from the results of the two previous steps after a couple of minimizations on the parameters $\epsilon_{t}$ and $\epsilon_{x}$. In this case, we can send $\epsilon_{t}$ to zero and then minimize over $\epsilon_{x}$ to get

$$
e(T) \leq e(0)+2\left\{\left|v_{0}\right|_{\left.T V\left(\mathbb{R}^{d}\right)\right)}+\left|u_{0}\right|_{T V\left(\mathbb{R}^{d}\right)}\right\}^{1 / 2}\left\{T\|\nu(u)\|\left|u_{0}\right|_{T V\left(\mathbb{R}^{d}\right)}\right\}^{1 / 2},
$$

where $\|\nu(u)\|=\sup _{\substack{t^{\prime} \in(0, T) \\ x^{\prime} \in \mathbb{R}^{d}}} \nu\left(u\left(t^{\prime}, x^{\prime}\right)\right)$. 


\section{A NEW APPROACH FOR OBTAINING A PRIORI ERROR ESTIMATES}

We next illustrate the main steps of the new approach. (The results stated below will be proven rigorously in $\S 5$.)

a. Choosing the entropy dissipation form $E(u, v ; \tau)$. We pick the form $E(u, v ; \tau)$ trying to capture in it all the information about the definition of the solution $u$. Thus, in our particular case, we pick $E(u, v ; \tau)$ as follows:

$$
E(u, v ; \tau)=\int_{0}^{\tau} \int_{\mathbb{R}^{d}} \Theta_{\tau}(u, v(t, x) ; t, x) d x d t
$$

where

$$
\Theta_{\tau}(u, c ; t, x)=\int_{0}^{\tau} \int_{\mathbb{R}^{d}} U^{\prime}\left(u\left(t^{\prime}, x^{\prime}\right)-c\right) L(u)\left(t^{\prime}, x^{\prime}\right) \varphi d x^{\prime} d t^{\prime}
$$

where $U$ and $\varphi$ are as in Kuznetsov's approach, and

$$
L(u) \equiv u_{t^{\prime}}+\nabla_{x^{\prime}} \cdot f(u)-\nabla_{x^{\prime}} \cdot\left(\nu(u) \nabla_{x^{\prime}} u\right) .
$$

Note that with this choice, we have $E(u, v ; \tau)=0$ for every function $v$ since $L(u)=$ 0 .

b. The 'dissipative' and 'divergence' parts of $E(u, v ; \tau)$. In this step, we try to identify the part of the form $E(u, v ; \tau)$ that will ensure the correct production of entropy dissipation. We call such a part, the 'dissipative' part of $E(u, v ; \tau)$ and we denote it by $E_{\text {diss }}(u, v ; \tau)$. Our objective is to be able to write

$$
E(u, v ; \tau)=-E_{\mathrm{diss}}(u, v ; \tau)+E_{\mathrm{div}}(u, v ; \tau),
$$

where the 'dissipative' part was chosen in such a way that the remaining part (which usually has a 'divergence' form), $E_{\text {div }}(u, v ; \tau)$, could be treated without having to use the entropy production properties of the scheme.

In this case, we have

$$
\begin{aligned}
& E_{\mathrm{diss}}(u, v ; \tau)=\int_{0}^{\tau} \int_{\mathbb{R}^{d}} \int_{0}^{\tau} \int_{\mathbb{R}^{d}}\left\{U^{\prime \prime}(u-v) \nu(u)\left|\nabla_{x^{\prime}} u\right|^{2}\right\} \varphi d x^{\prime} d t^{\prime} d x d t \\
& E_{\mathrm{div}}(u, v ; \tau)=\int_{0}^{\tau} \int_{\mathbb{R}^{d}} \int_{0}^{\tau} \int_{\mathbb{R}^{d}} D I V(u, v) \varphi d x^{\prime} d t^{\prime} d x d t
\end{aligned}
$$

where

$$
D I V(u, v)=(U(u-v))_{t^{\prime}}+\nabla_{x^{\prime}} \cdot\left(F(u, v)-\nu(u) \nabla_{x^{\prime}} U(u-v)\right) .
$$

c. Finding the dual form $E_{\text {div }}^{\star}(u, v ; \tau)$ and the error term $T_{\operatorname{err}}(u, v ; \tau)$. Next, we find a dual form $E_{\text {div }}^{\star}(u, v ; \tau)$ and an error term $T_{\operatorname{err}}(u, v ; \tau)$ such that

$$
E_{\text {div }}(u, v ; \tau)=-E_{\text {div }}^{\star}(u, v ; \tau)+T_{\text {err }}(u, v ; \tau) .
$$


In this case, the dual form is found as in the Kuznetsov approach. The error term is exactly the same. In this way, we have

$$
\begin{aligned}
e(\tau)-e(0) & \sim T_{\mathrm{err}}(u, v ; \tau) \\
& =E(u, v ; \tau)+E_{\mathrm{div}}^{\star}(u, v ; \tau)-E_{\mathrm{diss}}(u, v ; \tau) \\
& =E_{\mathrm{div}}^{\star}(u, v ; \tau)-E_{\mathrm{diss}}(u, v ; \tau),
\end{aligned}
$$

where the dual form is, in this case,

$$
E_{\text {div }}^{\star}(u, v ; \tau)=\int_{0}^{\tau} \int_{\mathbb{R}^{d}} \int_{0}^{\tau} \int_{\mathbb{R}^{d}} T E(v, u) \varphi d x d t d x^{\prime} d t^{\prime},
$$

where

$$
T E(v, u)=U(v-u)_{t}+\nabla_{x} F(v, u)-\nabla_{x} \cdot\left(U^{\prime}(u-v) \nu(v) \nabla_{x} v\right),
$$

and where for the sake of simplicity we have assumed that $v$ is smooth. Note that the dual form $E_{\text {div }}^{\star}(u, v ; \tau)$, is nothing but the 'truncation error' for the entropy/entropy flux pair $U / F$. This is our main motivation for the choice of $E(u, v ; \tau)$.

d. Finding a lower bound of the error term $T_{\operatorname{err}}(u, v ; \tau)$. Next, we want to obtain a lower bound of the error term $T_{\operatorname{err}}(u, v ; \tau)$ that is independent of the regularity properties of $u$. In the case under consideration, we obtain the following inequality

$$
\begin{aligned}
T_{\mathrm{err}}(u, v ; \tau) \geq & W(\tau) e(\tau)+\int_{0}^{\tau} w(\tau-t) e(t) d t \\
& -W(\tau) e(0)-\int_{0}^{\tau} w(t) e(t) d t \\
& -4 W(\tau)\left|v_{0}\right|_{T V\left(\mathbb{R}^{d}\right)}\left\{\epsilon_{x}+\left\|f^{\prime}(v)\right\| \epsilon_{t}\right\} .
\end{aligned}
$$

Note that there is no term that needs to be controlled by using regularity properties of $u$.

e. Finding an upper bound for the error $e(T)$. The price we have to pay for this advantage is to solve a sort of Gronwall-Volterra inequality. Indeed, in this case, after putting together the results of the previous steps, we obtain the following inequality:

$$
\begin{aligned}
W(\tau) e(\tau)+\int_{0}^{\tau} w(\tau-t) e(t) d t \leq & W(\tau) e(0)+\int_{0}^{\tau} w(t) e(t) d t \\
& +4 W(t)\left|v_{0}\right|_{T V\left(\mathbb{R}^{d}\right)}\left\{\epsilon_{x}+\left\|f^{\prime}(v)\right\| \epsilon_{t}\right\} \\
& +E^{\star}(u, v ; \tau)-E_{\mathrm{diss}}(u, v ; \tau),
\end{aligned}
$$

which can be solved to give the following inequality

$$
\begin{aligned}
e(T) \leq & 2 e(0)+8\left(\epsilon_{x}+\epsilon_{t}\left\|f^{\prime}(v)\right\|\right)\left|v_{0}\right|_{T V(\mathbb{R})} \\
& +2 \sup _{0 \leq t \leq T}\left\{E_{\text {div }}^{\star}(u, v ; t) / W(t)\right\} \\
& -2 \inf _{0 \leq t \leq T}\left\{E_{\text {diss }}(u, v ; t) / W(t)\right\} .
\end{aligned}
$$


f. Finding an upper bound for the dual form $E^{\star}(u, v ; t)$ and the dissipation form $E_{\text {diss }}(u, v ; \tau)$. At this point, to get an error estimate we only have to obtain estimates of the dual and the dissipation forms. The new feature we introduce in this paper is that it is possible to estimate the dual form $E^{\star}(u, v ; t)$ without taking into account the entropy dissipation properties of the scheme (which, roughly speaking, have been 'transferred' into the dissipation form $\left.E_{\text {diss }}(u, v ; \tau)\right)$ and without having to use the regularity of its approximate solution $u$. The estimate of the dual form depends only on the regularity properties of the entropy solution $v$.

Indeed, in our case, we get

$$
\begin{aligned}
& E_{\text {diss }}(u, v ; \tau) \geq 0, \\
& E_{\text {div }}^{\star}(u, v ; \tau) \leq E_{\text {reg }}(u, v ; \tau) .
\end{aligned}
$$

We want to emphasize that it is possible to estimate the form $E_{\mathrm{reg}}(u, v ; \tau)$ without using regularity properties of $u$ by performing a couple of simple integration by parts. To see this, set $V(u, v)=\int_{v}^{u} \nu(s) U^{\prime}(s-v) d s$ and assume that the entropy solution $v$ is smooth for the sake of clarity. We have

$$
\begin{aligned}
E_{\mathrm{reg}}(u, v ; \tau) & =\int_{0}^{\tau} \int_{\mathbb{R}} \int_{0}^{\tau} \int_{\mathbb{R}} \Delta_{x^{\prime}} V(u, v) \varphi\left(t, x, t^{\prime}, x^{\prime}\right) d x d t d x^{\prime} d t^{\prime} \\
& =\int_{0}^{\tau} \int_{\mathbb{R}} \int_{0}^{\tau} \int_{\mathbb{R}} V(u, v) \Delta_{x^{\prime}} \varphi\left(t, x, t^{\prime}, x^{\prime}\right) d x d t d x^{\prime} d t^{\prime} \\
& =-\int_{0}^{\tau} \int_{\mathbb{R}} \int_{0}^{\tau} \int_{\mathbb{R}} V(u, v) \nabla_{x} \cdot \nabla_{x^{\prime}} \varphi\left(t, x, t^{\prime}, x^{\prime}\right) d x d t d x^{\prime} d t^{\prime} \\
& =\int_{0}^{\tau} \int_{\mathbb{R}} \int_{0}^{\tau} \int_{\mathbb{R}} U^{\prime}(u-v) \nu(v) \nabla_{x} v \nabla_{x^{\prime}} \varphi\left(t, x, t^{\prime}, x^{\prime}\right) d x d t d x^{\prime} d t^{\prime} \\
& \leq \frac{2}{\epsilon_{x}} \tau\left|v_{0}\right|_{T V(\mathbb{R})}\|\nu(v)\| W(\tau) .
\end{aligned}
$$

If $v$ is not smooth, a similar estimate holds with $\|\nu(v)\|$ replaced by $\left\|\nu_{v}\right\|$; see Theorem 4.1 .

g. Getting the error estimate. This step is essentially identical to step 2 f. In this case, we get

$$
\|u(T)-v(T)\|_{L^{1}(\mathbb{R})} \leq 2\left\|u_{0}-v_{0}\right\|_{L^{1}\left(\mathbb{R}^{d}\right)}+4\left|v_{0}\right|_{T V(\mathbb{R})}\left\{8 T\left\|\nu_{v}\right\|\right\}^{1 / 2} .
$$

Before considering the first application of the above approach, let us briefly discuss the main differences between Kuznetsov's approach and the approach we have proposed:

(i) The choice of the form $E(u, v ; \tau)$. Kuznetsov picks $E(u, v ; \tau)$ as the original Kruškov form. We construct $E(u, v ; \tau)$ directly from the numerical scheme under consideration. This might give more flexibility in inserting the characteristics of the scheme into the form $E(u, v ; \tau)$.

(ii) The choice of $U$ and $\varphi$. Although we have not done this in the above steps, it is possible to use arbitrary even entropy functions $U$. This gives greater flexibility and allows to treat cases impossible to treat with the standard Kružkov entropy $U(w-c)=|w-c|$; see [1,2,3], [5], and [7]. Also, the choice of $\varphi$ does not have to be reduced to the one displayed in $\S 2 \mathrm{a}$. (This will be exploited in future work.) 
(iii) The modulus of continuity in time of $u$ is not required. The way in which the lower bound for the error term $T_{\text {err }}(u, v ; \tau)$ is obtained is completely different for Kuznetsov's approach. It is possible to avoid having to use the modulus of continuity in time of the approximation $u$, by a sort of Gronwall-Volterra inequality; see [5] and [7].

(iv) No regularity properties of $u$ is needed to get error estimates. We have shown how to estimate the dual form $E^{\star}(u, v ; \tau)$, or, equivalently, Kuznetsov's form $E_{\mathrm{reg}}(u, v ; \tau)$ in terms of the entropy solution only.

\section{A first applichtion: The model problem}

As a first application of the general theory of a priori error estimates, we obtain an upper bound between the entropy solution $v$ of (1.1) in the one-dimensional case $d=1$, and the solution $u$, in the sense of $\mathrm{Vol}^{\prime}$ pert and Hudjaev [27], of the parabolic initial value problem

$$
\begin{array}{ll}
u_{t}+\nabla \cdot f(u)-\nabla \cdot(\nu(u) \nabla u)=0 & \text { in }(0, T) \times \mathbb{R}, \\
u(0)=u_{0} & \text { on } \mathbb{R} .
\end{array}
$$

We include this result here because solutions of the above Cauchy problem mimic the behavior of several numerical schemes and so the estimate can be considered to be a continuous model of the estimate for the approximate solutions. Moreover, it is very simple to illustrate the approach displayed in $\S 3$ in this case since the function $u$ is smooth. Finally, we want to strongly emphasize that, against widespread belief, error estimates for nonlinear scalar conservation laws can be obtained without having to obtain regularity properties of the approximate solution.

Thus, the following result can be proven, see $\S 5$, assuming only that $u$ is a strong solution of (4.1) and that the viscosity coefficient $\nu(u)$ is positive. No estimate of the modulus of continuity in time, or estimate of the total variation in space, or estimate of the uniform norm of $u$ is explicitly used; only the standard regularity properties of the entropy solution $v$ are used. Note how the bound for the error is written solely in terms of the entropy solution $v$.

Theorem 4.1. Suppose that the nonlinear viscosity coefficient $\nu$ is positive on the range of $u$. Let $u$ be the solution of the parabolic problem (4.1) and let $v$ be the entropy solution of the conservation law (1.1) (with $d=1$ ), where $v_{0} \in$ $L^{\infty}(\mathbb{R}) \cap B V(\mathbb{R}) ;$ set $\mathcal{R}\left(v_{0}\right)=\left[\inf _{x \in \mathbb{R}} v_{0}(x), \sup _{x \in \mathbb{R}} v_{0}(x)\right]$. Then

$$
\|u(T)-v(T)\|_{L^{1}(\mathbb{R})} \leq 2\left\|u_{0}-v_{0}\right\|_{L^{1}(\mathbb{R})}+4\left|v_{0}\right|_{T V(\mathbb{R})}\left\{8 T\left\|\nu_{v}\right\|\right\}^{1 / 2}
$$

where

$$
\left\|\nu_{v}\right\|=\sup _{w \in \mathcal{R}\left(v_{0}\right)} \nu(w) .
$$

When the entropy solution has a finite number of discontinuity curves on each compact set of $(0, T) \times \mathbb{R}$, we can take

$$
\left\|\nu_{v}\right\|=\sup _{\substack{t \in(0, \tau) \\ x \in \mathbb{R}}} \nu(v(t, x-0), v(t, x+0)),
$$

where

$$
\nu\left(v^{-}, v^{+}\right)=\frac{1}{v^{+}-v^{-}} \int_{v^{-}}^{v^{+}} \nu(s) d s .
$$


It is well known that the entropy solution can display a finite number of discontinuity curves. For example, the number of discontinuity curves of the entropy solution of the Riemann problem is not bigger than one plus the number of inflection points of the nonlinearity $f$; see [20]. See also the recent work by Tadmor and Tassa [25] on the piecewise smoothness of entropy solutions for (1.1).

To end this section, let us stress the point that if we only assume that the solution $u \in \mathcal{C}^{0}\left(0, T ; L^{1}(\mathbb{R})\right)$, we can take advantage of the special structure of the parabolic regularization term in (4.1a) to get the following estimate:

$$
\|u(T)-v(T)\|_{L^{1}(\mathbb{R})} \leq\left\|u_{0}-v_{0}\right\|_{L^{1}(\mathbb{R})}+\left|v_{0}\right|_{T V(\mathbb{R})}\left\{8 T\left\|\nu_{v}\right\|\right\}^{1 / 2} .
$$

However, we have chosen not to present this 'improved' result since we want the proof of Theorem 4.1 to serve as a simplified model of the (much more technical) proof of the corresponding result (Theorem 6.1) for the discrete approximate solution given by the fully discrete Engquist-Osher scheme. In fact, if we do not discretize in time but only in space, we can still use the abovementioned trick, but it is not possible to use it anymore for the fully discrete case.

\section{Proof of Theorem 4.1}

In this section, we prove Theorem 4.1. To do so, we follow each of the steps of the new approach to a priori error estimates described in $\S 3$.

a. Choosing the entropy dissipation form $E(u, v ; \tau)$. In this case, we use the choice described in $\S 3$ a with $L(u)=u_{t^{\prime}}+f(u)_{x^{\prime}}-\left(\nu(u) u_{x^{\prime}}\right)_{x^{\prime}}$. In other words, we take

$$
E(u, v ; \tau)=\int_{0}^{\tau} \int_{\mathbb{R}} \Theta_{\tau}(u, v(t, x) ; t, x) d x d t
$$

where

$$
\Theta_{\tau}(u, c ; t, x)=\int_{0}^{\tau} \int_{\mathbb{R}} \Psi\left(u\left(t^{\prime}, x^{\prime},\right), c\right) \varphi d x^{\prime} d t^{\prime},
$$

and

$$
\Psi(u, c)=U^{\prime}(u-c)\left\{u_{t^{\prime}}+f(u)_{x^{\prime}}-\left(\nu(u) u_{x^{\prime}}\right)_{x^{\prime}}\right\} .
$$

We take $U$ and $\varphi$ as in Kuznetsov's original approach, but this time, we must minimize the constants of the right-hand side of the inequality resulting from the resolution of the Gronwall-Volterra inequality in $\S 3 \mathrm{e}$. To do so, we take a sequence of functions $w$, satisfying $(2.1)$ and being nonincreasing on $(0,1)$, that converges pointwise to

$$
\chi(s)= \begin{cases}\frac{1}{2} & \text { for }|s|<1, \\ 0 & \text { otherwise. }\end{cases}
$$

In what follows, we denote this limit process by ' $w \rightarrow \chi$ '. Note that we have

$$
\lim _{w \rightarrow \chi}|w|_{T V(\mathbb{R})}=|\chi|_{T V(\mathbb{R})}=1 .
$$

b. The "divergence" and "dissipative" parts of $E\left(u_{h}, v ; \tau\right)$. The first step in obtaining the entropy inequality by the well-known vanishing viscosity method, [14], consists in rewriting $\Psi(u, c)$ as follows:

$$
\Psi(u, c)=D I V(u, c)+\operatorname{LRED}(u, c),
$$


where

$$
\begin{aligned}
& \operatorname{DIV}(u, c)=\left\{(U(u-c))_{t^{\prime}}+\left(F(u, c)-\nu(u) U(u-c)_{x^{\prime}}\right)_{x^{\prime}}\right\}, \\
& \operatorname{LRED}(u, c)=\left\{U^{\prime \prime}(u-c) \nu(u)\left(u_{x^{\prime}}\right)^{2}\right\} .
\end{aligned}
$$

The first term is in divergence form and can thus be called the "divergence" part of $\Psi(u, c)$. The second term can be called the "local rate of entropy dissipation." This simple, but useful, decomposition of $\Psi(u, c)$ allows us to rewrite $E(u, v ; \tau)$ as the sum $E_{\text {div }}(u, v ; \tau)+E_{\text {diss }}(u, v ; \tau)$, where the "divergence" part $E_{\text {div }}(u, v ; \tau)$ is

$$
E_{\operatorname{div}}(u, v ; \tau)=\int_{0}^{\tau} \int_{\mathbb{R}} \int_{0}^{\tau} \int_{\mathbb{R}} D I V(u, v) \varphi\left(t, x, t^{\prime}, x^{\prime}\right) d x d t,
$$

and the "dissipative" part $E_{\text {diss }}(u, v ; \tau)$ is

$$
E_{\mathrm{diss}}(u, v ; \tau)=\int_{0}^{\tau} \int_{\mathbb{R}} \int_{0}^{\tau} \int_{\mathbb{R}} \operatorname{LRED}(u, v) \varphi\left(t, x, t^{\prime}, x^{\prime}\right) d x d t .
$$

We have the following immediate result.

Proposition 5.1. Suppose that the nonlinear viscosity coefficient $\nu$ is positive on the range of $u$. Then we have

$$
E_{\text {diss }}(u, v ; \tau) \geq 0 \text {. }
$$

c. The dual form $E_{\text {div }}^{\star}(u, v ; \tau)$ and the error term $T_{\text {err }}(u, v ; \tau)$. In this step, we perform a simple integration by parts and strongly use the structure of the auxiliary function $\varphi$. Setting $V(u, v)=\int_{v}^{u} \nu(s) U^{\prime}(s-v) d s$, and taking into account that $\varphi_{t}=-\varphi_{t^{\prime}}$ and $\varphi_{x}=-\varphi_{x^{\prime}}$, we have

$$
\begin{aligned}
& E_{\mathrm{div}}(u, v ; \tau)=\int_{0}^{\tau} \int_{\mathbb{R}} \int_{0}^{\tau} \int_{\mathbb{R}}\left\{(U(u-v))_{t^{\prime}}+\left(F(u, v)-V(u, v)_{x^{\prime}}\right)_{x^{\prime}}\right\} \\
& \cdot \varphi\left(t, x, t^{\prime}, x^{\prime}\right) d x^{\prime} d t^{\prime} d x d t \\
& =\int_{0}^{\tau} \int_{\mathbb{R}} \int_{0}^{\tau} \int_{\mathbb{R}}\left\{-U(u-v) \varphi_{t^{\prime}}\left(t, x, t^{\prime}, x^{\prime}\right)-F(u, v) \varphi_{x^{\prime}}\left(t, x, t^{\prime}, x^{\prime}\right)\right. \\
& \left.-V(u, v) \varphi_{x^{\prime} x^{\prime}}\left(t, x, t^{\prime}, x^{\prime}\right)\right\} d x^{\prime} d t^{\prime} d x d t \\
& +\int_{0}^{\tau} \int_{\mathbb{R}} \int_{\mathbb{R}} U(u(\tau)-v) \varphi\left(t, x, \tau, x^{\prime}\right) d x^{\prime} d x d t \\
& -\int_{0}^{\tau} \int_{\mathbb{R}} \int_{\mathbb{R}} U\left(u_{0}-v\right) \varphi\left(t, x, 0, x^{\prime}\right) d x^{\prime} d x d t \\
& =\int_{0}^{\tau} \int_{\mathbb{R}} \int_{0}^{\tau} \int_{\mathbb{R}}\left\{U(u-v) \varphi_{t}\left(t, x, t^{\prime}, x^{\prime}\right)+F(u, v) \varphi_{x}\left(t, x, t^{\prime}, x^{\prime}\right)\right. \\
& \left.+V(u, v) \varphi_{x x^{\prime}}\left(t, x, t^{\prime}, x^{\prime}\right)\right\} d x^{\prime} d t^{\prime} d x d t \\
& +\int_{0}^{\tau} \int_{\mathbb{R}} \int_{\mathbb{R}} U(u(\tau)-v) \varphi\left(t, x, \tau, x^{\prime}\right) d x^{\prime} d x d t \\
& -\int_{0}^{\tau} \int_{\mathbb{R}} \int_{\mathbb{R}} U\left(u_{0}-v\right) \varphi\left(t, x, 0, x^{\prime}\right) d x^{\prime} d x d t .
\end{aligned}
$$


Setting

$$
\begin{aligned}
E_{\mathrm{div}}^{\star}(u, v ; \tau)= & \int_{0}^{\tau} \int_{\mathbb{R}} \int_{0}^{\tau} \int_{\mathbb{R}}\left\{-U(u-v) \varphi_{t}\left(t, x, t^{\prime}, x^{\prime}\right)-F(u, v) \varphi_{x}\left(t, x, t^{\prime}, x^{\prime}\right)\right. \\
& +\int_{0}^{\tau} \int_{\mathbb{R}} \int_{\mathbb{R}} U(u-v(\tau)) \varphi\left(\tau, x, t^{\prime}, x^{\prime}\right) d x d x^{\prime} d t^{\prime} \\
& -\int_{0}^{\tau} \int_{\mathbb{R}} \int_{\mathbb{R}} U\left(u-v_{0}\right) \varphi\left(0, x, t^{\prime}, x^{\prime}\right) d x d x^{\prime} d t^{\prime}
\end{aligned}
$$

we have

$$
\begin{aligned}
E_{\mathrm{div}}(u, v ; \tau)= & -E_{\mathrm{div}}^{\star}(u, v ; \tau) \\
& +\int_{0}^{\tau} \int_{\mathbb{R}} \int_{\mathbb{R}} U(u(\tau)-v) \varphi\left(t, x, \tau, x^{\prime}\right) d x^{\prime} d x d t \\
& +\int_{0}^{\tau} \int_{\mathbb{R}} \int_{\mathbb{R}} U(u-v(\tau)) \varphi\left(\tau, x, t^{\prime}, x^{\prime}\right) d x d x^{\prime} d t^{\prime} \\
& -\int_{0}^{\tau} \int_{\mathbb{R}} \int_{\mathbb{R}} U\left(u_{0}-v\right) \varphi\left(t, x, 0, x^{\prime}\right) d x^{\prime} d x d t \\
& -\int_{0}^{\tau} \int_{\mathbb{R}} \int_{\mathbb{R}} U\left(u-v_{0}\right) \varphi\left(0, x, t^{\prime}, x^{\prime}\right) d x d x^{\prime} d t^{\prime} \\
\equiv & -E_{\mathrm{div}}^{\star}(u, v ; \tau)+T_{\mathrm{err}}(u, v ; \tau) .
\end{aligned}
$$

d. A lower bound of the error term $T_{\text {err }}(u, v ; \tau)$.

Proposition 5.2 [7]. We have

$$
\begin{aligned}
T_{\mathrm{err}}(u, v ; \tau) \geq & W(\tau) e(\tau)+\int_{0}^{\tau} w_{\epsilon_{t}}(\tau-t) e(t) d t \\
& -W(\tau) e(0)-\int_{0}^{\tau} w_{\epsilon_{t}}(t) e(t) d t \\
& -4 W(\tau)\left\{\left\|f^{\prime}(v)\right\| \epsilon_{t}+\epsilon_{x}\right\}\left|v_{0}\right|_{T V(\mathbb{R})}
\end{aligned}
$$

For the sake of completeness, we give a proof of this result below.

Proof. We see, from $\S 5 \mathrm{c}$, that we can write $T_{\text {err }}=T_{1}+T_{2}+T_{3}+T_{4}$, with the obvious notation. Let us start by estimating $T_{1}$. Since

$$
\begin{aligned}
U\left(u\left(\tau, x^{\prime}\right)-v(t, x)\right)= & U\left(u\left(\tau, x^{\prime}\right)-v\left(\tau, x^{\prime}\right)\right) \\
& +\left\{U\left(u\left(\tau, x^{\prime}\right)-v(\tau, x)\right)-U\left(u\left(\tau, x^{\prime}\right)-v\left(\tau, x^{\prime}\right)\right)\right\} \\
& +\left\{U\left(u\left(\tau, x^{\prime}\right)-v(t, x)\right)-U\left(u\left(\tau, x^{\prime}\right)-v(\tau, x)\right)\right\} \\
\geq & U\left(u\left(\tau, x^{\prime}\right)-v\left(\tau, x^{\prime}\right)\right) \\
& -\left|v(\tau, x)-v\left(\tau, x^{\prime}\right)\right|-|v(t, x)-v(\tau, x)|
\end{aligned}
$$


we get

$$
\begin{aligned}
T_{1}= & \int_{0}^{\tau} \int_{\mathbb{R}} \int_{\mathbb{R}} U\left(u\left(\tau, x^{\prime}\right)-v(t, x)\right) \varphi\left(t, x, \tau, x^{\prime}\right) d x^{\prime} d x d t \\
\geq & \int_{0}^{\tau} \int_{\mathbb{R}} \int_{\mathbb{R}} U\left(u\left(\tau, x^{\prime}\right)-v\left(\tau, x^{\prime}\right)\right) \varphi\left(t, x, \tau, x^{\prime}\right) d x^{\prime} d x d t \\
& -\int_{0}^{\tau} \int_{\mathbb{R}} \int_{\mathbb{R}}\left|v\left(\tau, x^{\prime}\right)-v\left(t, x^{\prime}\right)\right| \varphi\left(t, x, \tau, x^{\prime}\right) d x^{\prime} d x d t \\
& -\int_{0}^{\tau} \int_{\mathbb{R}} \int_{\mathbb{R}} \mid\left(v\left(\tau, x^{\prime}\right)-v(\tau, x) \mid \varphi\left(t, x, \tau, x^{\prime}\right) d x^{\prime} d x d t\right. \\
\geq & W(\tau) \int_{\mathbb{R}} U\left(u\left(\tau, x^{\prime}\right)-v\left(\tau, x^{\prime}\right)\right) d x^{\prime}-W(\tau)\left\{\left\|f^{\prime}(v)\right\| \epsilon_{t}+\epsilon_{x}\right\}\left|v_{0}\right|_{T V(\mathbb{R})} .
\end{aligned}
$$

Now, let us consider the second term, $T_{2}$. Proceeding as for the first term, we get

$$
\begin{aligned}
T_{2}= & \int_{0}^{\tau} \int_{\mathbb{R}} \int_{\mathbb{R}} U\left(u\left(t^{\prime}, x^{\prime}\right)-v(\tau, x)\right) \varphi\left(\tau, x, t^{\prime}, x^{\prime}\right) d x d x^{\prime} d t^{\prime} \\
\geq & \int_{0}^{\tau} \int_{\mathbb{R}} \int_{\mathbb{R}} U\left(u\left(t^{\prime}, x^{\prime}\right)-v\left(t^{\prime}, x^{\prime}\right)\right) \varphi\left(\tau, x, t^{\prime}, x^{\prime}\right) d x d x^{\prime} d t^{\prime} \\
& -\int_{0}^{\tau} \int_{\mathbb{R}} \int_{\mathbb{R}}\left|u\left(t^{\prime}, x^{\prime}\right)-v\left(\tau, x^{\prime}\right)\right| \varphi\left(\tau, x, t^{\prime}, x^{\prime}\right) d x d x^{\prime} d t^{\prime} \\
& -\int_{0}^{\tau} \int_{\mathbb{R}} \int_{\mathbb{R}}\left|u\left(\tau, x^{\prime}\right)-v(\tau, x)\right| \varphi\left(\tau, x, t^{\prime}, x^{\prime}\right) d x d x^{\prime} d t^{\prime} \\
\geq & \int_{0}^{\tau} w_{\epsilon_{t}}\left(\tau-t^{\prime}\right)\left\{\int_{\mathbb{R}} U\left(u\left(t^{\prime}, x^{\prime}\right)-v\left(t^{\prime}, x^{\prime}\right)\right) d x^{\prime}\right\} d t^{\prime} \\
& -W(\tau)\left\{\left\|f^{\prime}(v)\right\| \epsilon_{t}+\epsilon_{x}\right\}\left|v_{0}\right|_{T V(\mathbb{R})} .
\end{aligned}
$$

To estimate $T_{3}$ and $T_{4}$ we proceed in a similar way. We obtain

$$
\begin{aligned}
T_{3} \geq & -W(\tau) \int_{\mathbb{R}} U\left(u\left(0, x^{\prime}\right)-v\left(0, x^{\prime}\right)\right) d x^{\prime} \\
& -W(\tau)\left\{\left\|f^{\prime}(v)\right\| \epsilon_{t}+\epsilon_{x}\right\} \mid v_{0} \|_{T V(\mathbb{R})}, \\
T_{4} \geq & -\int_{0}^{\tau} w_{\epsilon_{t}}\left(t^{\prime}\right) \int_{\mathbb{R}} U\left(\left(v\left(t^{\prime}, x^{\prime}\right)-v\left(t^{\prime}, x^{\prime}\right)\right) d x^{\prime}\right. \\
& -W(\tau)\left\{\left\|f^{\prime}(v)\right\| \epsilon_{t}+\epsilon_{x}\right\}\left|v_{0}\right|_{T V(\mathbb{R})} .
\end{aligned}
$$

This completes the proof.

\section{e. The approximation inequality.}

Proposition 5.3 (The approximation inequality). We have

$$
\begin{aligned}
e(T) \leq & 2 e(0)+8\left(\epsilon_{x}+\epsilon_{t}\left\|f^{\prime}(v)\right\|\right)\left|v_{0}\right|_{T V(\mathbb{R})} \\
& +2 \lim _{w \rightarrow \chi} \sup _{0 \leq t \leq T}\left\{E_{\text {div }}^{\star}(u, v ; t) / W(t)\right\} .
\end{aligned}
$$

To prove this proposition, we need the following auxiliary result. 
Lemma 5.4. Let $\theta:[0, T] \mapsto \mathbb{R}^{+}$be a nonnegative, measurable function such that for all $\tau \in[0, T]$

$$
W^{\infty}(\tau) \theta(\tau)+\int_{0}^{\tau} w_{\epsilon_{t}}^{\infty}(\tau-t) \theta(t) d t \leq W^{\infty}(\tau) C+\int_{0}^{\tau} w_{\epsilon_{t}}^{\infty}(t) \theta(t) d t
$$

where $w^{\infty}=\chi$. Then, for $\tau \in[0, T]$,

$$
\theta(\tau) \leq 2 C
$$

Proof. If $\tau \leq \epsilon_{t}$, we have for $t \in[0, \tau]$ that $w_{\epsilon_{t}}^{\infty}(\tau-t)=w_{\epsilon_{t}}^{\infty}(t) \equiv 1 / 2 \epsilon_{t}$, and so

$$
\theta(\tau) \leq C
$$

To estimate $\theta(\tau)$ for $\tau \geq \epsilon_{t}$, we rewrite the inequality satisfied by $\theta(\tau)$ as follows:

$$
\begin{aligned}
\theta(\tau) & \leq C+\int_{0}^{\tau}\left\{w_{\epsilon_{t}}^{\infty}(t)-w_{\epsilon_{t}}^{\infty}(\tau-t)\right\} \theta(t) d t / W^{\infty}(\tau) \\
& \leq C+\int_{0}^{\epsilon_{t}}\left\{w_{\epsilon_{t}}^{\infty}(t)-w_{\epsilon_{t}}^{\infty}(\tau-t)\right\} \theta(t) d t / W^{\infty}(\tau),
\end{aligned}
$$

since for $t>\epsilon_{t}, w_{\epsilon_{t}}^{\infty}(t)-w_{\epsilon_{t}}^{\infty}(\tau-t)=-w_{\epsilon_{t}}^{\infty}(\tau-t) \leq 0$ and since $\theta(t) \geq 0$. Since $\theta(t) \leq C$ for $t \in\left(0, \epsilon_{t}\right)$, this implies that

$$
\begin{aligned}
\theta(\tau) & \leq\left\{1+\int_{0}^{\epsilon_{t}}\left\{w_{\epsilon_{t}}^{\infty}(t)-w_{\epsilon_{t}}^{\infty}(\tau-t)\right\} d t / W^{\infty}(\tau)\right\} C \\
& \leq 2 C
\end{aligned}
$$

This completes the proof.

We are now ready to prove Proposition 5.3.

Proof of Proposition 5.3. We have, from Propositions 5.2 and 5.1,

$$
\begin{aligned}
W(\tau) e(\tau)+\int_{0}^{\tau} w_{\epsilon_{t}}(\tau-t) e(t) d t \leq & W(\tau) e(0)+\int_{0}^{\tau} w_{\epsilon_{t}}(t) e(t) d t \\
& +4 W(\tau)\left\{\left\|f^{\prime}(v)\right\| \epsilon_{t}+\epsilon_{x}\right\}\left|v_{0}\right|_{T V(\mathbb{R})} \\
& +E_{\text {div }}^{\star}(u, v ; \tau)
\end{aligned}
$$

Passing to the limit in $w$, we obtain, for $\tau \in[0, T]$,

$$
\begin{aligned}
W^{\infty}(\tau) e(\tau)+\int_{0}^{\tau} w_{\epsilon_{t}}^{\infty}(\tau-t) e(t) d t \leq & W^{\infty}(\tau) e(0)+\int_{0}^{\tau} w_{\epsilon_{t}}^{\infty}(t) e(t) d t \\
& +4 W^{\infty}(\tau)\left\{\left\|f^{\prime}(v)\right\| \epsilon_{t}+\epsilon_{x}\right\}\left|v_{0}\right|_{T V(\mathbb{R})} \\
& +W^{\infty}(\tau) \lim _{w \rightarrow \chi} \sup _{t \in(0, T)}\left\{E_{\operatorname{div}}^{\star}(u, v ; t) / W(t)\right\} .
\end{aligned}
$$

We can now apply Lemma 5.4 with $\theta=e$, and

$$
C=e(0)+4\left\{\left\|f^{\prime}(v)\right\| \epsilon_{t}+\epsilon_{x}\right\}\left|v_{0}\right|_{T V(\mathbb{R})}+\lim _{w \rightarrow \chi} \sup _{t \in(0, T)}\left\{E_{\operatorname{div}}^{\star}(u, v ; t) / W(t)\right\} .
$$

This completes the proof. 
f. The estimate of $\lim _{w \rightarrow \chi} \sup _{t \in(0, T)}\left\{E_{\text {div }}^{\star}(u, v ; t) / W(t)\right\}$.

Proposition 5.5. For $U(w)=|w|$, we have,

$$
\lim _{w \rightarrow \chi} \sup _{0 \leq t \leq T}\left\{E_{\text {div }}^{\star}(u, v ; t) / W(t)\right\} \leq \frac{2}{\epsilon_{x}} T\left|v_{0}\right|_{T V(\mathbb{R})}\left\|\nu_{v}\right\|,
$$

where $\left\|\nu_{v}\right\|$ is defined as in Theorem 4.1 .

To prove this proposition, we need the four auxiliary results that follow.

Lemma 5.6. We have

$$
-F(u, v)+F(v, u)=\int_{v}^{u}\left\{f^{\prime}((u+v) / 2)-f^{\prime}(s)\right\}\left\{U^{\prime}(s-u)+U^{\prime}(s-v)\right\} d s
$$

for any even function $U$ such that $U(0)=0$. In particular, for $U(w)=|w|$, we have

$$
-F(u, v)+F(v, u)=0 .
$$

The proof of this result is straightforward; see, e.g., [7].

Lemma 5.7. Set $V(u, v)=\int_{v}^{u} \nu(s) U^{\prime}(s-v) d s$. Then, for $U(w)=|w|$, we have,

$$
\left|\frac{1}{v^{+}-v^{-}}\left(V\left(u, v^{+}\right)-V\left(u, v^{-}\right)\right)\right| \leq \frac{1}{v^{+}-v^{-}} \int_{v^{-}}^{v^{+}} \nu(s) d s .
$$

This inequality follows easily from the definition of $V(u, v)$ and that of $U$.

Lemma 5.8. We have

$$
\int_{0}^{\tau} \sup _{x \in \mathbb{R}} D(t, x) d t \leq \frac{2|w|_{T V(\mathbb{R})}}{\epsilon_{x}} \tau W(\tau)
$$

where

$$
D(t, x)=\int_{0}^{\tau} \int_{\mathbb{R}}\left|\varphi_{x^{\prime}}\left(t, x, t^{\prime}, x^{\prime}\right)\right| d x^{\prime} d t^{\prime}
$$

Proof. By the definition of $\varphi$ in $\S 2 \mathrm{a}$,

$$
D(t, x) \leq \frac{|w|_{T V(\mathbb{R})}}{\epsilon_{x}} \int_{0}^{\tau}\left|w_{\epsilon_{t}}\left(t-t^{\prime}\right)\right| d t^{\prime},
$$

and so, after simple algebraic manipulations, we get

$$
\begin{aligned}
\int_{0}^{\tau} \sup _{x \in \mathbb{R}} D(t, x) d t & \leq \frac{|w|_{T V(\mathbb{R})}}{\epsilon_{x}} \int_{0}^{\tau} \int_{0}^{\tau} w_{\epsilon_{t}}\left(t-t^{\prime}\right) d t^{\prime} d t \\
& =\frac{2|w|_{T V(\mathbb{R})}}{\epsilon_{x}} \int_{0}^{\tau}(\tau-s) w_{\epsilon_{t}}(s) d s \\
& \leq \frac{2|w|_{T V(\mathbb{R})}}{\epsilon_{x}} \tau W(\tau) .
\end{aligned}
$$

This completes the proof. 
Lemma 5.9. There exists a sequence $\left\{v_{j}\right\}_{j \in \mathbb{N}}$ of functions in $\mathcal{C}^{0}\left(0, T ; L^{1}(\mathbb{R})\right)$ that converges to $v$ in $L^{\infty}\left(0, T ; L^{1}(\mathbb{R})\right)$ such that

$$
\begin{aligned}
& \left|v_{j}(t)\right|_{T V(\mathbb{R})} \leq\left|v_{0}\right|_{T V(\mathbb{R})} \text { for } j \in \mathbb{N}, t \in(0, T), \\
& \inf _{x \in \mathbb{R}} v_{j}(t, x) \geq \inf _{x \in \mathbb{R}} v_{0}(x) \quad \text { for } j \in \mathbb{N}, t \in(0, T), \\
& \sup _{x \in \mathbb{R}} v_{j}(t, x) \leq \sup _{x \in \mathbb{R}} v_{0}(x) \quad \text { for } j \in \mathbb{N}, t \in(0, T) .
\end{aligned}
$$

Proof. Take $v_{j}(t, x)=\left(\delta_{j} * v(t)\right)(x)$, where $\delta_{j}(y)=j \delta(j y)$ and $\delta$ satisfies the conditions $(2.1)$. Since $v \in \mathcal{C}^{0}\left(0, T ; L^{1}(\mathbb{R})\right)$, the sequence $\left\{v_{j}\right\}_{j \in \mathbb{N}}$ is a sequence in $\mathcal{C}^{0}\left(0, T ; L^{1}(\mathbb{R})\right)$ that converges to $v$ in $L^{\infty}\left(0, T ; L^{1}(\mathbb{R})\right)$. Finally, the result follows from the following standard inequalities:

$$
\begin{array}{ll}
\left|v_{j}(t)\right|_{T V(\mathbb{R})} \leq|v(t)|_{T V(\mathbb{R})} & \text { for } j \in \mathbb{N}, t \in(0, T), \\
\inf _{x \in \mathbb{R}} v_{j}(t, x) \geq \inf _{x \in \mathbb{R}} v(t, x) & \text { for } j \in \mathbb{N}, t \in(0, T), \\
\sup _{x \in \mathbb{R}} v_{j}(t, x) \leq \sup _{x \in \mathbb{R}} v(t, x) & \text { for } j \in \mathbb{N}, t \in(0, T),
\end{array}
$$

and from the following properties of the entropy solution $v$ :

$$
\begin{array}{ll}
|v(t)|_{T V(\mathbb{R})} \leq\left|v_{0}\right|_{T V(\mathbb{R})} & \text { for } t \in(0, T), \\
\inf _{x \in \mathbb{R}} v(t, x) \geq \inf _{x \in \mathbb{R}} v_{0}(x) & \text { for } t \in(0, T), \\
\sup _{x \in \mathbb{R}} v(t, x) \leq \sup _{x \in \mathbb{R}} v_{0}(x) & \text { for } t \in(0, T) .
\end{array}
$$

This completes the proof.

We are now ready to prove Proposition (5.5).

Proof of Proposition 5.5. Let us begin by proving the result in the case in which the entropy solution $v$ is smooth everywhere except at most on a single curve $\mathcal{C}=\{(x(t), t): t \in(0, \tau)\}$.

By the definition of the form $E_{\text {div }}^{\star}(u, v ; t)$, the definition of the entropy solution, and Lemma 5.6 , we have

$$
E_{\mathrm{div}}^{\star}(u, v ; \tau) \leq-\int_{0}^{\tau} \int_{\mathbb{R}} \int_{0}^{\tau} \int_{\mathbb{R}} V(u, v) \varphi_{x x^{\prime}}\left(t, x, t^{\prime}, x^{\prime}\right) d x d t d x^{\prime} d t^{\prime}
$$

After a couple of simple integration by parts, we can rewrite (5.2) as follows:

$$
E_{\mathrm{div}}^{\star}(u, v ; \tau) \leq \int_{0}^{\tau} \int_{\mathbb{R}} \Psi\left(t^{\prime}, x^{\prime}\right) d x^{\prime} d t^{\prime},
$$

where

$$
\begin{aligned}
\Psi\left(t^{\prime}, x^{\prime}\right)=\int_{0}^{\tau}\left\{\int_{-\infty}^{x(t)} \partial_{v} V(u, v) v_{x} \varphi_{x^{\prime}} d x\right. & +\frac{[V]}{[v]}[v] \varphi_{x^{\prime}} \\
& \left.+\int_{x(t)}^{\infty} \partial_{v} V(u, v) v_{x} \varphi_{x^{\prime}} d x\right\} d t
\end{aligned}
$$


Here, $[G]$ denotes the jump of the function $G(v)$ at the point $(x(t), t)$, that is, the value $G(v(t, x(t)+0))-G(v(t, x(t)-0))$.

Taking absolute values and using Lemma 5.7, we obtain

$$
\left|\Psi\left(t^{\prime}, x^{\prime}\right)\right| \leq\left\|\nu_{v}\right\| \int_{0}^{\tau}\left\{\int_{-\infty}^{x(t)}\left|v_{x}\right|\left|\varphi_{x^{\prime}}\right| d x+|[v]|\left|\varphi_{x^{\prime}}\right|+\int_{x(t)}^{\infty}\left|v_{x}\right|\left|\varphi_{x^{\prime}}\right| d x\right\} d t .
$$

Finally, by using the quantity $D$ defined in Lemma 5.8 , we obtain

$$
\begin{aligned}
E_{\text {div }}^{\star}(u, v ; \tau) & \leq\left\|\nu_{v}\right\| \int_{0}^{\tau}\left\{\int_{-\infty}^{x(t)}\left|v_{x}\right| D(t, x) d x+|[v]| D(t, x(t))\right. \\
& \left.\quad+\int_{x(t)}^{\infty}\left|v_{x}\right| D(t, x) d x\right\} d t \\
& \leq\left\|\nu_{v}\right\||v|_{L^{\infty}(0, T ; T V(\mathbb{R}))} \int_{0}^{\tau} \sup _{x \in \mathbb{R}} D(t, x) d t \\
& \leq \frac{2|w|_{T V(\mathbb{R})}}{\epsilon_{x}} \tau\left|v_{0}\right|_{T V(\mathbb{R})}\left\|\nu_{v}\right\| W(\tau),
\end{aligned}
$$

by Lemma 5.8 and since $|v|_{L^{\infty}(0, T ; T V(\mathbb{R}))} \leq\left|v_{0}\right|_{T V(\mathbb{R})}$. The result follows from (5.1).

This proves the result for the case in which the entropy solution $v$ is smooth everywhere except at most on a single curve $\mathcal{C}=\{(x(t), t): t \in(0, \tau)\}$. The same technique can be used to prove the estimate in the case the entropy solution has a finite number of discontinuity curves in each compact of $(0, T) \times \mathbb{R}$.

Let us now consider the general case. By the inequality (5.2), Lemma 5.9, and since the function $V(u, v)$ is Lipschitz in $v$ by Lemma 5.7 , we have

$$
\begin{aligned}
E_{\mathrm{div}}^{\star}(u, v ; \tau) & \leq-\int_{0}^{\tau} \int_{\mathbb{R}} \int_{0}^{\tau} \int_{\mathbb{R}} V(u, v) \varphi_{x x^{\prime}}\left(t, x, t^{\prime}, x^{\prime}\right) d x d t d x^{\prime} d t^{\prime} \\
& =\lim _{j \rightarrow \infty}-\int_{0}^{\tau} \int_{\mathbb{R}} \int_{0}^{\tau} \int_{\mathbb{R}} V\left(u, v_{j}\right) \varphi_{x x^{\prime}}\left(t, x, t^{\prime}, x^{\prime}\right) d x d t d x^{\prime} d t^{\prime} .
\end{aligned}
$$

Since $v_{j}$ does not have discontinuities, we have, by (5.3),

$$
\begin{aligned}
E_{\mathrm{div}}^{\star}(u, v ; \tau) & \leq \lim _{j \rightarrow \infty} \frac{2|w|_{T V(\mathbb{R})}}{\epsilon_{x}} \tau\left|v_{j}\right|_{L^{\infty}(0, T ; T V(\mathbb{R}))}\left\|\nu_{v_{j}}\right\| W(\tau) \\
& \leq \frac{2|w|_{T V(\mathbb{R})}}{\epsilon_{x}} \tau\left|v_{0}\right|_{T V(\mathbb{R})}\left\|\nu_{v}\right\| W(\tau),
\end{aligned}
$$

by Lemma 5.9. This completes the proof.

g. Proof of the error estimate. From $\S 5 \mathrm{~d}$ and $\S 5 \mathrm{e}$, we have that, for $U(w)=$ $|w|$,

$$
\begin{aligned}
\|u(T)-v(T)\|_{L^{1}(\mathbb{R})} \leq & 2\left\|u_{0}-v_{0}\right\|_{L^{1}(\mathbb{R})}+8\left(\epsilon_{x}+\epsilon_{t}\left\|f^{\prime}(v)\right\|\right)\left|v_{0}\right|_{T V(\mathbb{R})} \\
& +\frac{4}{\epsilon_{x}} T\left|v_{0}\right|_{T V(\mathbb{R})}\left\|\nu_{v}\right\|
\end{aligned}
$$

Letting $\epsilon_{t}$ go to zero and minimizing over $\epsilon_{x}$, we get

$$
\|u(T)-v(T)\|_{L^{1}(\mathbb{R})} \leq 2\left\|u_{0}-v_{0}\right\|_{L^{1}(\mathbb{R})}+4\left|v_{0}\right|_{T V(\mathbb{R})}\left\{8 T\left\|\nu_{v}\right\|\right\}^{1 / 2} .
$$

This completes the proof of Theorem 4.1. 


\section{A second Application: The Engquist-Osher scheme}

As a second application, we consider the problem of obtaining a priori error estimates for the well-known Engquist-Osher scheme. For the sake of simplicity, we use uniform grids. Let $\Delta t$ and $\Delta x$ be two given positive numbers. Set $x_{j}=$ $j \Delta x, x_{j+1 / 2}=\left(x_{j}+x_{j+1}\right) / 2$ and $t^{n}=n \Delta t$.

The approximate solution

$$
u(t, x)=u_{j}^{n} \quad \text { for }(t, x) \in\left[t^{n}, t^{n+1}\right) \times\left(x_{j-1 / 2}, x_{j+1 / 2}\right),
$$

defined by the Engquist-Osher scheme, is given by the solution of the following equations:

$$
\left(u_{j}^{n+1}-u_{j}^{n}\right) / \Delta t+\left(f^{E O}\left(u_{j}^{n}, u_{j+1}^{n}\right)-f^{E O}\left(u_{j-1}^{n}, u_{j}^{n}\right)\right) / \Delta x=0, \quad n \in \mathbb{N}, j \in \mathbb{Z},
$$

where the numerical flux $f^{E O}$ is given by

$$
f^{E O}(a, b)=f^{+}(a)+f^{-}(b),
$$

where

$$
\begin{aligned}
& f^{+}(a)=\int^{a} \max \left\{f^{\prime}(s), 0\right\} d s, \\
& f^{-}(b)=\int^{a} \min \left\{f^{\prime}(s), 0\right\} d s
\end{aligned}
$$

and the initial condition is $u(t=0)=u_{0}=P_{\Delta x}\left(v_{0}\right)$, where $P_{\Delta x}$ is some operator from $B V(\mathbb{R})$ to the space of functions which are constant on the intervals $\left(x_{j-1 / 2}, x_{x+1 / 2}\right)$, for $j \in \mathbb{Z}$.

The model equation of the Engquist-Osher scheme [10] is

$$
u_{t}+(f(u))_{x}-\left(\nu^{E O}(u) u_{x}\right)_{x}=0
$$

where

$$
\nu^{E O}(u)=\frac{\Delta x}{2}\left\{\left|f^{\prime}(u)\right|-\frac{\Delta t}{\Delta x}\left(f^{\prime}(u)\right)^{2}\right\}
$$

Since we expect the approximate solution of the Engquist-Osher scheme to behave like the solution of (4.1) with the above viscosity, an error estimate similar to the one of Theorem 4.1 should hold provided that the viscosity coefficient is positive, which is true if

$$
\frac{\Delta t}{\Delta x}\left\|f^{\prime}(u)\right\| \leq 1
$$

Our next result shows that this is indeed the case. Note that the bound of the error is given solely in terms of the entropy solution $v$.

Theorem 6.1. Let the Courant-Friedrichs-Levy condition (6.2) be satisfied. Let $u$ be the piecewise constant approximate solution given by the Engquist-Osher scheme 
defined above, and let $v$ be the entropy solution of $(1.1)$ (with $d=1$ ) where $v_{0} \in$ $L^{\infty}(\mathbb{R}) \cap B V(\mathbb{R}) ;$ set $\mathcal{R}\left(v_{0}\right)=\left[\inf _{x \in \mathbb{R}} v_{0}(x), \sup _{x \in \mathbb{R}} v_{0}(x)\right]$. Then

$$
\begin{aligned}
\left\|u\left(t^{N}\right)-v\left(t^{N}\right)\right\|_{L^{1}(\mathbb{R})} \leq & 2\left\|u_{0}-v_{0}\right\|_{L^{1}(\mathbb{R})}+4\left|v_{0}\right|_{T V(\mathbb{R})}\left\{8 t^{N}\left\|\nu_{v}\right\|\right\}^{1 / 2} \\
& +\left|v_{0}\right|_{T V(\mathbb{R})}\left\{b_{1}(\Delta x)^{3 / 4}+b_{2} \Delta x\right\}
\end{aligned}
$$

where

$$
\left\|\nu_{v}\right\|=\sup _{w \in \mathcal{R}\left(v_{0}\right)} \nu^{E O}(w)
$$

for $\nu^{E O}$ given by (6.1). When the entropy solution has a finite number of discontinuities on each compact set of $(0, T) \times \mathbb{R}$, we can take

$$
\left\|\nu_{v}\right\|=\sup _{\substack{t \in(0, \tau) \\ x \in \mathbb{R}}} \nu^{E O}(v(t, x-0), v(t, x+0))
$$

and

$$
\nu^{E O}\left(v^{-}, v^{+}\right)=\frac{\Delta x}{2}\left(\frac{1}{v^{+}-v^{-}} \int_{v^{-}}^{v^{+}}\left|f^{\prime}(s)\right| d s-\frac{\Delta t}{\Delta x}\left\{\frac{f\left(v^{+}\right)-f\left(v^{-}\right)}{v^{+}-v^{-}}\right\}^{2}\right) .
$$

The coefficients $b_{1}$ and $b_{2}$ are bounded functions that depend on the following quantities:

$$
\mathrm{cfl}=\left\|f^{\prime}(v)\right\| \Delta t / \Delta x, \quad \zeta=\left\|f^{\prime}(v)\right\| \Delta x /\left\|\nu_{v}\right\|, \quad \kappa=\left\{t^{N}\left\|\nu_{v}\right\| / \Delta x\right\}^{1 / 2} .
$$

Note that if we drop the asymptotically unimportant terms containing the coefficients $b_{1}$ and $b_{2}$, this result is identical with its 'continuous version', Theorem 4.1 (with a suitably defined viscosity). Note also that

$$
\frac{\Delta x}{2} \frac{1}{v^{+}-v^{-}} \int_{v^{-}}^{v^{+}}\left|f^{\prime}(s)\right| d s
$$

is exactly equal to the so-called viscosity produced by the Engquist-Osher flux. The negative viscosity

$$
-\frac{\Delta t}{2}\left\{\frac{f\left(v^{+}\right)-f\left(v^{-}\right)}{v^{+}-v^{-}}\right\}^{2}
$$

is produced by the explicit nature of the scheme. As is well known, the stability condition (6.2) ensures that the (total) viscosity of the scheme, $\nu^{E O}\left(v^{-}, v^{+}\right)$, is nonnegative.

The above result is proven without explicitly using any regularity properties of the approximate solution; instead, we only use the regularity properties of the entropy solution. The only properties of the Engquist-Osher scheme that we explicitly use are that the scheme satisfies a (i) local entropy inequality which (ii) has conservation form and (iii) uses a two-point splitting entropy flux. Any other scheme with these properties satisfies a result similar to Theorem 6.1. In this paper, in order to better illustrate the new approach to a priori error estimates, we have restricted ourselves to a simple model scheme in a simple setting (the one-dimensional case and uniform grids) for the sake of simplicity and clarity. A generalization of Theorem 6.1 to the $d$-dimensional case and a general triangulation will be treated in a forthcoming paper. 


\section{Proof of Theorem 6.1}

The proof of Theorem 6.1 is, essentially, a "discrete" version of the proof of Theorem 4.1.

a. The choice of $E\left(u, v ; t^{N}\right)$. Following what was done in the continuous case in $\S 5 \mathrm{a}$, we pick the form $E\left(u, v ; t^{N}\right)$ as follows:

$$
E\left(u_{h}, v ; t^{N}\right)=\int_{0}^{t^{N}} \int_{\mathbb{R}} \sum_{n=0}^{N-1} \sum_{j \in \mathbb{Z}} \Psi_{j}^{n}(v(t, x)) \phi\left(t, x, t^{n+1}, x_{j}\right) \Delta x \Delta t d x d t,
$$

where

$$
\Psi_{j}^{n}(c)=U^{\prime}\left(u_{j}^{n}-c\right)\left\{\left(u_{j}^{n+1}-u_{j}^{n}\right) / \Delta t+\left(\Delta_{-} f^{+}\left(u_{j}^{n}\right)+\Delta_{+} f^{-}\left(u_{j}^{n}\right)\right) / \Delta x\right\},
$$

and

$$
\phi\left(t, x, t^{\prime}, x_{j}\right)=\frac{1}{\Delta x} \int_{x_{j-1 / 2}}^{x_{j+1 / 2}} \varphi\left(t, x, t^{\prime}, s\right) d s .
$$

As in the continuous case, the entropy $U$ is the Kružkov entropy. The function $\varphi=\varphi\left(t, x, t^{\prime}, x^{\prime}\right)$ is taken as follows:

$$
\varphi=w_{\epsilon_{t}}\left(t-t^{\prime}\right) \eta_{\epsilon_{x}}(x-x), \quad(x, t),\left(x^{\prime}, t^{\prime}\right) \in \mathbb{R} \times \mathbb{R}^{+},
$$

where $\epsilon_{t}$ and $\epsilon_{x}$ are two arbitrary positive numbers and

$$
w_{\epsilon_{t}}(s)=\frac{1}{\epsilon_{t}} w\left(\frac{s}{\epsilon_{t}}\right), \quad \eta_{\epsilon_{x}}(s)=\frac{1}{\epsilon_{x}} \eta\left(\frac{s}{\epsilon_{x}}\right),
$$

for any $s \in \mathbb{R}$. The functions $w$ and $\eta$ are smooth functions satisfying the properties (2.1). As in the continuous case, we take a sequence of functions $w$ converging to the function $\chi$. However, we do not want to do the same thing with the functions $\eta$ since, as will become clear later in the proof, we need to estimate the total variation of the second derivative of $\eta$. Thus, we take a sequence of functions $\eta$ converging to the function

$$
\chi_{\epsilon}(x)= \begin{cases}(1+\epsilon) / 2 & \text { for }|x| \leq(1-\epsilon) /(1+\epsilon), \\ (1+\epsilon)^{2}(1-|x|) / 4 \epsilon & \text { for }|x| \in[(1-\epsilon) /(1+\epsilon), 1] \\ 0 & \text { elsewhere }\end{cases}
$$

It is easy to verify that we can find a sequence of functions $\eta$ such that

$$
\begin{aligned}
& \lim _{\eta \rightarrow \chi_{\epsilon}}|\eta|_{T V(\mathbb{R})}=\left|\chi_{\epsilon}\right|_{T V(\mathbb{R})}=1+\epsilon, \\
& \lim _{\eta \rightarrow \chi_{\epsilon}}\left|\eta^{\prime}\right|_{T V(\mathbb{R})}=\left|\chi_{\epsilon}^{\prime}\right|_{T V(\mathbb{R})}=2+\epsilon+1 / \epsilon .
\end{aligned}
$$

b. The "divergence" and "dissipative" parts of $E\left(u_{h}, v ; t^{N}\right)$. In this step, we rewrite the form $E\left(u_{h}, v ; t^{N}\right)$ as the sum of its "divergence" and "dissipative" parts. 
Proposition 7.1 (The "divergence" and "dissipative" parts of $\left.E\left(u_{h}, v ; t^{N}\right)\right)$. We have

$$
E\left(u_{h}, v ; t^{N}\right)=E_{\operatorname{div}}\left(u_{h}, v ; t^{N}\right)+E_{\text {diss }}\left(u_{h}, v ; t^{N}\right) .
$$

The "divergence" part $E_{\mathrm{div}}\left(u_{h}, v ; t^{N}\right)$ is given by

$$
E_{\mathrm{div}}\left(u_{h}, v ; t^{N}\right)=\int_{0}^{t^{N}} \int_{\mathbb{R}} \sum_{n=0}^{N-1} \sum_{j \in \mathbb{Z}} D I V_{j}^{n}(v(t, x)) \phi\left(t, x, t^{n+1}, x_{j}\right) \Delta x \Delta t d x d t,
$$

where

$$
D I V_{j}^{n}(c)=\left(U\left(u_{j}^{n+1}-c\right)-U\left(u_{j}^{n}-c\right)\right) / \Delta t+\left(\Delta_{-} F^{+}\left(u_{j}^{n}, c\right)+\Delta_{+} F^{-}\left(u_{j}^{n}, c\right)\right) / \Delta x,
$$

where

$$
F^{ \pm}(u, c)=\int_{c}^{u}\left(f^{ \pm}\right)^{\prime}(s) U^{\prime}(s-c) d s
$$

The "dissipative" part $E_{\mathrm{diss}}\left(u_{h}, v ; t^{N}\right)$ is given by

$$
E_{\mathrm{diss}}\left(u_{h}, v ; t^{N}\right)=\int_{0}^{t^{N}} \int_{\mathbb{R}} \sum_{n=0}^{N-1} \sum_{j \in \mathbb{Z}} L R E D_{j}^{n}(v(t, x)) \phi\left(t, x, t^{n+1}, x_{j}\right) \Delta x \Delta t d x d t,
$$

where the local rate of entropy dissipation $\operatorname{LRE} D_{j}^{n}(c)$ is given by

$$
\begin{aligned}
\operatorname{LRED}_{j}^{n}(c)= & \frac{1}{\Delta t} \int_{u_{j}^{n+1}}^{u_{j}^{n}}\left(h\left(u_{j}^{n}\right)-h(s)\right) U^{\prime \prime}(s-c) d s \\
& +\frac{1}{\Delta x} \int_{u_{j}^{n+1}}^{u_{j-1}^{n}}\left(f^{+}\left(u_{j-1}^{n}\right)-f^{+}(s)\right) U^{\prime \prime}(s-c) d s \\
& +\frac{1}{\Delta x} \int_{u_{j}^{n+1}}^{u_{j+1}^{n}}\left(-f^{-}\left(u_{j+1}^{n}\right)+f^{-}(s)\right) U^{\prime \prime}(s-c) d s,
\end{aligned}
$$

where $h(s)=s-\frac{\Delta t}{\Delta x}\left(f^{+}(s)-f^{-}(s)\right)$.

Proof. By definition, we have

$$
E\left(u_{h}, v ; t^{N}\right)=\int_{0}^{t^{N}} \int_{\mathbb{R}} \sum_{n=0}^{N-1} \sum_{j \in \mathbb{Z}} \Psi_{j}^{n}(v(t, x)) \phi\left(t, x, t^{n+1}, x_{j}\right) \Delta x \Delta t d x d t,
$$

where

$$
\begin{aligned}
\Psi_{j}^{n}(c) & =U^{\prime}\left(u_{j}^{n}-c\right)\left\{\left(u_{j}^{n+1}-u_{j}^{n}\right) / \Delta t+\left(\Delta_{-} f^{+}\left(u_{j}^{n}\right)+\Delta_{+} f^{-}\left(u_{j}^{n}\right)\right) / \Delta x\right\} \\
& =U^{\prime}\left(u_{j}^{n}-c\right)\left(u_{j}^{n+1}-u_{j}^{n}\right) / \Delta t+U^{\prime}\left(u_{j}^{n}-c\right) \Delta_{-} f^{+}\left(u_{j}^{n}\right) / \Delta x \\
& \quad+U^{\prime}\left(u_{j}^{n}-c\right) \Delta_{+} f^{-}\left(u_{j}^{n}\right) / \Delta x \\
& \equiv \Psi_{1}(c)+\Psi_{2}(c)+\Psi_{3}(c) .
\end{aligned}
$$

Note that $\Psi_{j}^{n}(c)$ is a discrete version of the expression $\Psi(u, c)$ defined in $\S 5$ a.

To obtain the result, we use the following simple identity: 


$$
U^{\prime}(a-c)(g(b)-g(a))=G(b, c)-G(a, c)-\int_{a}^{b}(g(b)-g(s)) U^{\prime \prime}(s-c) d s,
$$

where

$$
G(b, c)=\int_{c}^{b} g^{\prime}(s) U^{\prime}(s-c) d s .
$$

Thus, taking $g(s)=s$, we get

$$
\Psi_{1}(c)=\left(U\left(u_{j}^{n+1}-c\right)-U\left(u_{j}^{n}-c\right)\right) / \Delta t-\frac{1}{\Delta t} \int_{u_{j}^{n}}^{u_{j}^{n+1}}\left(u_{j}^{n+1}-s\right) U^{\prime \prime}(s-c) d s .
$$

Next, taking $g(s)=f^{-}(s)$, we obtain

$$
\Psi_{2}(c)=\left(\Delta_{-} F^{+}\left(u_{j}^{n}, c\right)\right) / \Delta x+\frac{1}{\Delta x} \int_{u_{j}^{n}}^{u_{j-1}^{n}}\left(f^{+}\left(u_{j-1}^{n}\right)-f^{+}(s)\right) U^{\prime \prime}(s-c) d s .
$$

Finally, taking $g(s)=f^{+}(s)$, we obtain

$$
\Psi_{3}(c)=\left(\Delta_{-} F^{-}\left(u_{j}^{n}, c\right)\right) / \Delta x-\frac{1}{\Delta x} \int_{u_{j}^{n}}^{u_{j+1}^{n}}\left(f^{-}\left(u_{j+1}^{n}\right)-f^{-}(s)\right) U^{\prime \prime}(s-c) d s .
$$

Gathering the last three expressions, we have

$$
\Psi_{j}^{n}(c)=D I V_{j}^{n}(c)+L R E D_{j}^{n}(c),
$$

where

$$
D I V_{j}^{n}(c)=\left(U\left(u_{j}^{n+1}-c\right)-U\left(u_{j}^{n}-c\right)\right) / \Delta t+\left(\Delta_{-} F^{+}\left(u_{j}^{n}, c\right)+\Delta_{+} F^{-}\left(u_{j}^{n}, c\right)\right) / \Delta x,
$$

and

$$
\begin{aligned}
\operatorname{LRED} D_{j}^{n}(c)= & -\frac{1}{\Delta t} \int_{u_{j}^{n}}^{u_{j}^{n+1}}\left(u_{j}^{n+1}-s\right) U^{\prime \prime}(s-c) d s \\
& +\frac{1}{\Delta x} \int_{u_{j}^{n}}^{u_{j-1}^{n}}\left(f^{+}\left(u_{j-1}^{n}\right)-f^{+}(s)\right) U^{\prime \prime}(s-c) d s \\
& -\frac{1}{\Delta x} \int_{u_{j}^{n}}^{u_{j+1}^{n}}\left(f^{-}\left(u_{j+1}^{n}\right)-f^{-}(s)\right) U^{\prime \prime}(s-c) d s \\
= & \left.-\frac{1}{\Delta t} \int_{u_{j}^{n}}^{u_{j}^{n+1}}\left\{u_{j}^{n+1}-\frac{\Delta t}{\Delta x}\left(f^{+}\left(u_{j-1}^{n}\right)-f^{-}\left(u_{j+1}^{n}\right)\right)-h(s)\right)\right\} \\
& +\frac{1}{\Delta x} \int_{u_{j}^{n+1}}^{u_{j-1}^{n}}\left(f^{+}\left(u_{j-1}^{n}\right)-f^{+}(s)\right) U^{\prime \prime}(s-c) d s \\
& +\frac{1}{\Delta x} \int_{u_{j}^{n+1}}^{u_{j+1}^{n}}\left(-f^{-}\left(u_{j+1}^{n}\right)+f^{-}(s)\right) U^{\prime \prime}(s-c) d s .
\end{aligned}
$$


Using the definition of $u_{j}^{n+1}$, we get

$$
\begin{aligned}
\operatorname{LRED}_{j}^{n}(c)= & \left.\frac{1}{\Delta t} \int_{u_{j}^{n+1}}^{u_{j}^{n}}\left\{u_{j}^{n}-\frac{\Delta t}{\Delta x}\left(f^{+}\left(u_{j}^{n}\right)-f^{-}\left(u_{j}^{n}\right)\right)-h(s)\right)\right\} U^{\prime \prime}(s-c) d s \\
& +\frac{1}{\Delta x} \int_{u_{j}^{n+1}}^{u_{j-1}^{n}}\left(f^{+}\left(u_{j-1}^{n}\right)-f^{+}(s)\right) U^{\prime \prime}(s-c) d s \\
& +\frac{1}{\Delta x} \int_{u_{j}^{n+1}}^{u_{j+1}^{n}}\left(-f^{-}\left(u_{j+1}^{n}\right)+f^{-}(s)\right) U^{\prime \prime}(s-c) d s .
\end{aligned}
$$

This completes the proof.

Note that $D I V_{j}^{n}$ and $L R E D_{j}^{n}(c)$ are the discrete versions of the terms

$$
D I V(u, c)=\left\{(U(u-c))_{t}+\left(F(u, c)-\nu(u) U(u-c)_{x}\right)_{x}\right\}
$$

and

$$
\operatorname{LRED}(u, c)=\left\{U^{\prime \prime}(u-c) \nu(u)\left(u_{x}\right)^{2}\right\}
$$

respectively. To see this, consider that $u_{i}^{n}$ means $u\left(t^{n+1}, x_{i}\right)$ for arbitrary $i \in \mathbb{Z}$. Expanding around $\left(t^{n+1}, x_{j}\right)$, we get

$$
\begin{aligned}
D I V_{j}^{n}(c) & =\left\{(U(u-c))_{t}+\left(F(u, c)-\nu(u) U(u-c)_{x}\right)_{x}\right\}+\text { h. o.t., } \\
L R E D_{j}^{n}(c) & =\left\{U^{\prime \prime}(u-c) \nu(u)\left(u_{x}\right)^{2}\right\}+\text { h. o.t., }
\end{aligned}
$$

where

$$
\nu(u)=\frac{\Delta x}{2}\left\{\left|f^{\prime}(u)\right|-\frac{\Delta t}{\Delta x}\left(f^{\prime}(u)\right)^{2}\right\}
$$

as expected. This simple computation shows that $\nu(u)$ is nonnegative if $\frac{\Delta t}{\Delta x}\left|f^{\prime}(u)\right|$ $\leq 1$. The same result holds for the local rate of entropy dissipation.

Proposition 7.2 (Nonnegativity of the local rate of entropy dissipation). The local rate of entropy dissipation $\operatorname{LRE} D_{j}^{n}(c)$ is nonnegative if $\frac{\Delta t}{\Delta x}\left|f^{\prime}(s)\right| \leq 1$.

Proof. From the expression of the local entropy dissipation $L R E D_{j}^{n}(c)$ in Proposition 7.1, it is clear that $L R E D_{j}^{n}(c) \geq 0$ if the functions $h(s),-f^{-}(s)$ and $f^{+}(s)$ are nondecreasing functions of $s$. The functions $-f^{-}$and $f^{+}$satisfy that property by construction. It is very simple to verify that under the condition $\frac{\Delta t}{\Delta x}\left|f^{\prime}(u)\right| \leq 1$, the function $h$ is nondecreasing. This completes the proof.

Corollary 7.3 (Nonnegativity of the dissipative part $\left.E_{\mathrm{diss}}\left(u_{h}, v ; t^{N}\right)\right)$. If the hypotheses of Proposition 7.2 are satisfied, then $E_{\mathrm{diss}}\left(u_{h}, v ; t^{N}\right) \geq 0$.

c. The dual form $E_{\text {div }}^{\star}\left(u_{h}, v ; t^{N}\right)$ and the error term $T_{\operatorname{err}}\left(u_{h}, v ; t^{N}\right)$. To obtain the dual form and the error term, we simply make a couple of integrations by parts and use the fact that $u\left(t^{\prime}, x^{\prime}\right)$ is equal to $u_{j}^{n}$ on the rectangle $\left[t^{n}, t^{n+1}\right) \times$ 
$\left(x_{j-1 / 2}, x_{j+1 / 2}\right)$. We use the following notation:

$$
|F|=\left(F^{+}-F^{-}\right) / 2 .
$$

Proposition 7.4. We have

$$
E_{\text {div }}\left(u_{h}, v ; t^{N}\right)=-E_{\text {div }}^{\star}\left(u_{h}, v ; t^{N}\right)+T_{\operatorname{err}}\left(u_{h}, v ; t^{N}\right),
$$

where

$$
\begin{aligned}
E_{\mathrm{div}}^{\star}\left(u_{h}, v ; t^{N}\right)= & -\int_{0}^{t^{N}} \int_{\mathbb{R}} \int_{0}^{t^{N}} \int_{\mathbb{R}} U\left(u\left(t^{\prime}, x^{\prime}\right)-v(t, x)\right) \varphi_{t}\left(t, x, t^{\prime}, x^{\prime}\right) d x d t d x^{\prime} d t^{\prime} \\
& +\int_{0}^{t^{N}} \int_{\mathbb{R}} \int_{\mathbb{R}} U\left(u\left(t^{\prime}, x^{\prime}\right)-v\left(t^{N}, x\right)\right) \varphi\left(t^{N}, x, t^{\prime}, x^{\prime}\right) d x d t d x^{\prime} \\
& -\int_{0}^{t^{N}} \int_{\mathbb{R}} \int_{\mathbb{R}} U\left(u\left(t^{\prime}, x^{\prime}\right)-v_{0}(x)\right) \varphi\left(t^{N}, x, t^{\prime}, x^{\prime}\right) d x d t d x^{\prime} \\
& -\sum_{n=0}^{N-1} \int_{\mathbb{R}} \int_{0}^{t^{N}} \int_{\mathbb{R}} \frac{1}{2 \Delta x}\left(\varphi\left(t, x+\Delta x, t^{n+1}, x^{\prime}\right)-\varphi\left(t, x-\Delta x, t^{n+1}, x^{\prime}\right)\right) \\
& \cdot F\left(u\left(t^{n}, x^{\prime}\right), v(t, x)\right) d x d t d x^{\prime} \Delta t \\
& +\sum_{n=0}^{N-1} \int_{\mathbb{R}} \int_{0}^{t^{N}} \int_{\mathbb{R}} \frac{1}{2 \Delta x}\left(\varphi\left(t, x+\Delta x, t^{n+1}, x^{\prime}\right)-2 \varphi\left(t, x, t^{n+1}, x^{\prime}\right)\right. \\
& +F\left(u\left(t^{n}, x^{\prime}\right), v(t, x)\right) \mid d x d t d x^{\prime} \Delta t,
\end{aligned}
$$

and

$$
\begin{aligned}
T_{\operatorname{err}}\left(u_{h}, v ; t^{N}\right)= & \int_{0}^{t^{N}} \int_{\mathbb{R}} \int_{\mathbb{R}} U\left(u\left(t^{N}, x^{\prime}\right)-v(t, x)\right) \varphi\left(t, x, t^{N}, x^{\prime}\right) d x^{\prime} d x d t \\
& +\int_{0}^{t^{N}} \int_{\mathbb{R}} \int_{\mathbb{R}} U\left(u\left(t^{\prime}, x^{\prime}\right)-v\left(t^{N}, x\right)\right) \varphi\left(t^{N}, x, t^{\prime}, x^{\prime}\right) d x d x^{\prime} d t^{\prime} \\
& -\int_{0}^{t^{N}} \int_{\mathbb{R}} \int_{\mathbb{R}} U\left(u\left(0, x^{\prime}\right)-v(t, x)\right) \varphi\left(t, x, 0, x^{\prime}\right) d x^{\prime} d x d t \\
& -\int_{0}^{t^{N}} \int_{\mathbb{R}} \int_{\mathbb{R}} U\left(u\left(t^{\prime}, x^{\prime}\right)-v_{0}(x)\right) \varphi\left(0, x, t^{\prime}, x^{\prime}\right) d x d x^{\prime} d t^{\prime} .
\end{aligned}
$$

Proof. After a standard integration by parts in time and another in space, we easily get

$$
E_{\mathrm{div}}\left(u_{h}, v ; t^{N}\right)=E_{\mathrm{div}, t}\left(u_{h}, v ; t^{N}\right)+E_{\mathrm{div}, x}\left(u_{h}, v ; t^{N}\right),
$$


where

$$
\begin{aligned}
E_{\mathrm{div}, t}\left(u_{h}, v ; t^{N}\right)= & \sum_{n=0}^{N-1} \sum_{j \in \mathbb{Z}} \int_{0}^{t^{N}} \int_{\mathbb{R}} U\left(u_{j}^{n}-v(t, x)\right) \\
& +\sum_{j \in \mathbb{Z}} \int_{0}^{t^{N}} \int_{\mathbb{R}} U\left(\phi\left(t, x, t^{n}, x_{j}\right)-\phi\left(t, x, t^{n+1}, x_{j}\right)\right) \Delta x d x d t \\
& -\sum_{j \in \mathbb{Z}} \int_{0}^{t^{N}} \int_{\mathbb{R}} U\left(u\left(0, x_{j}\right)-v(t, x)\right) \phi\left(t, x, t^{N}, x_{j}\right) \Delta x d x d t
\end{aligned}
$$

and

$$
\begin{aligned}
E_{\operatorname{div}, x}\left(u_{h}, v ; t^{N}\right)= & \sum_{n=0}^{N-1} \sum_{j \in \mathbb{Z}} \int_{0}^{t^{N}} \int_{\mathbb{R}}\left(\phi\left(t, x, t^{n+1}, x_{j}\right)-\phi\left(t, x, t^{n+1}, x_{j+1}\right)\right) \\
& \cdot F^{+}\left(u_{j}^{n}, v(t, x)\right) \Delta t d x d t \\
& +\sum_{n=0}^{N-1} \sum_{j \in \mathbb{Z}} \int_{0}^{t^{N}} \int_{\mathbb{R}}\left(\phi\left(t, x, t^{n+1}, x_{j-1}\right)-\phi\left(t, x, t^{n+1}, x_{j}\right)\right) \\
\cdot & \cdot F^{-}\left(u_{j}^{n}, v(t, x)\right) \Delta t d x d t
\end{aligned}
$$

Next, taking into account that $u\left(t^{\prime}, x^{\prime}\right)$ is equal to $u^{n}\left(x^{\prime}\right)$ on $\left[t^{n}, t^{n+1}\right)$, that $|F|=$ $F^{+}-F^{-}$, and noting that $F=F^{+}+F^{-}$, we can rewrite $E_{\operatorname{div}, t}\left(u_{h}, v ; t^{N}\right)$ and $E_{\text {div }, x}\left(u_{h}, v ; t^{N}\right)$ as follows:

$$
\begin{aligned}
E_{\mathrm{div}, t}\left(u_{h}, v ; t^{N}\right)= & -\int_{0}^{t^{N}} \sum_{j \in \mathbb{Z}} \int_{0}^{t^{N}} \int_{\mathbb{R}} U\left(u\left(t^{\prime}, x_{j}\right)-v(t, x)\right) \phi_{t^{\prime}}\left(t, x, t^{\prime}, x_{j}\right) d x d t \Delta x d t^{\prime} \\
& +\sum_{j \in \mathbb{Z}} \int_{0}^{t^{N}} \int_{\mathbb{R}} U\left(u\left(t^{N}, x_{j}\right)-v(t, x)\right) \phi\left(t, x, t^{N}, x_{j}\right) \Delta x d x d t \\
& -\sum_{j \in \mathbb{Z}} \int_{0}^{t^{N}} \int_{\mathbb{R}} U\left(u\left(0, x_{j}\right)-v(t, x)\right) \phi\left(t, x, 0, x_{j}\right) \Delta x d x d t
\end{aligned}
$$

and

$$
\begin{array}{r}
E_{\operatorname{div}, x}\left(u_{h}, v ; t^{N}\right)=\sum_{n=0}^{N-1} \sum_{j \in \mathbb{Z}} \int_{0}^{t^{N}} \int_{\mathbb{R}} \frac{1}{2}\left(\phi\left(t, x, t^{n+1}, x_{j-1}\right)-\phi\left(t, x, t^{n+1}, x_{j+1}\right)\right) \\
\cdot F\left(u_{j}^{n}, v(t, x)\right) \Delta t d x d t \\
-\sum_{n=0}^{N-1} \sum_{j \in \mathbb{Z}} \int_{0}^{t^{N}} \int_{\mathbb{R}} \frac{1}{2}\left(\phi\left(t, x, t^{n+1}, x_{j-1}\right)-2 \phi\left(t, x, t^{n+1}, x_{j}\right)\right. \\
\left.+\phi\left(t, x, t^{n+1}, x_{j+1}\right)\right)\left|F\left(u_{j}^{n}, v(t, x)\right)\right| \Delta t d x d t .
\end{array}
$$


Taking into account that $\phi_{t}=-\phi_{t^{\prime}}$, that $\phi\left(t, x, t^{\prime}, x_{j \pm 1}\right)=\phi\left(t, x \mp \Delta x, t^{\prime}, x_{j}\right)$, that $u\left(t^{\prime}, x^{\prime}\right)$ is equal to $u_{j}\left(t^{\prime}\right)$ on $\left(x_{j-1 / 2}, x_{j+1 / 2}\right)$, and that, by (7.1),

$$
\phi\left(t, x, t^{\prime}, x_{j}\right)=\frac{1}{\Delta x} \int_{x_{j-1 / 2}}^{x_{j+1 / 2}} \varphi\left(t, x, t^{\prime}, s\right) d s
$$

we get

$$
\begin{aligned}
E_{\mathrm{div}, t}\left(u_{h}, v ; t^{N}\right)= & \int_{0}^{t^{N}} \int_{\mathbb{R}} \int_{0}^{t^{N}} \int_{\mathbb{R}} U\left(u\left(t^{\prime}, x^{\prime}\right)-v(t, x)\right) \varphi_{t}\left(t, x, t^{\prime}, x^{\prime}\right) d x d t d x^{\prime} d t^{\prime} \\
& +\int_{\mathbb{R}} \int_{0}^{t^{N}} \int_{\mathbb{R}} U\left(u\left(t^{N}, x^{\prime}\right)-v(t, x)\right) \varphi\left(t, x, t^{N}, x^{\prime}\right) d x^{\prime} d x d t \\
& -\int_{\mathbb{R}} \int_{0}^{t^{N}} \int_{\mathbb{R}} U\left(u\left(0, x^{\prime}\right)-v(t, x)\right) \varphi\left(t, x, 0, x^{\prime}\right) d x^{\prime} d x d t
\end{aligned}
$$

and

$$
\begin{array}{r}
E_{\operatorname{div}, x}\left(u_{h}, v ; t^{N}\right)=\sum_{n=0}^{N-1} \int_{\mathbb{R}} \int_{0}^{t^{N}} \int_{\mathbb{R}} \frac{1}{2 \Delta x}\left(\varphi\left(t, x+\Delta x, t^{n+1}, x^{\prime}\right)-\varphi\left(t, x-\Delta x, t^{n+1}, x^{\prime}\right)\right) \\
\cdot F\left(u\left(t^{n}, x^{\prime}\right), v(t, x)\right) d x d t d x^{\prime} \Delta t \\
-\sum_{n=0}^{N-1} \int_{\mathbb{R}} \int_{0}^{t^{N}} \int_{\mathbb{R}} \frac{1}{2 \Delta x}\left(\varphi\left(t, x+\Delta x, t^{n+1}, x^{\prime}\right)-2 \varphi\left(t, x, t^{n+1}, x^{\prime}\right)\right. \\
\left.+\varphi\left(t, x-\Delta x, t^{n+1}, x^{\prime}\right)\right) \\
\cdot\left|F\left(u\left(t^{n}, x^{\prime}\right), v(t, x)\right)\right| d x d t d x^{\prime} \Delta t .
\end{array}
$$

Finally, by using the definition of the dual form, we can write

$$
\begin{aligned}
E_{\mathrm{div}}\left(u_{h}, v ; t^{N}\right)= & -E_{\mathrm{div}}^{\star}\left(u_{h}, v ; t^{N}\right) \\
& +\int_{\mathbb{R}} \int_{0}^{t^{N}} \int_{\mathbb{R}} U\left(u\left(t^{N}, x^{\prime}\right)-v(t, x)\right) \varphi\left(t, x, t^{N}, x^{\prime}\right) d x^{\prime} d x d t \\
& -\int_{\mathbb{R}} \int_{0}^{t^{N}} \int_{\mathbb{R}} U\left(u\left(0, x^{\prime}\right)-v(t, x)\right) \varphi\left(t, x, 0, x^{\prime}\right) d x^{\prime} d x d t \\
& +\int_{0}^{t^{N}} \int_{\mathbb{R}} \int_{0}^{t^{N}} \int_{\mathbb{R}} U\left(u\left(t^{\prime}, x^{\prime}\right)-v\left(t^{N}, x\right)\right) \varphi\left(t^{N}, x, t^{\prime}, x^{\prime}\right) d x d x^{\prime} d t^{\prime} \\
& -\int_{0}^{t^{N}} \int_{\mathbb{R}} \int_{\mathbb{R}} U\left(u\left(t^{\prime}, x^{\prime}\right)-v_{0}(x)\right) \varphi\left(t^{N}, x, t^{\prime}, x^{\prime}\right) d x d x^{\prime} d t^{\prime} .
\end{aligned}
$$

This completes the proof.

It is clear from the above proof, that the definition of $\phi\left(t, x, t^{\prime}, x_{j}\right)(7.1)$ allows us to have an error term $T_{\operatorname{err}}\left(u, v ; t^{N}\right)$ exactly equal to the error term of the continuous case. As a consequence, the next two steps are identical to the ones of the continuous case. 
d. A lower bound for the error term $T_{\operatorname{err}}\left(u, v ; t^{N}\right)$.

Proposition 7.5. We have

$$
\begin{aligned}
T_{\mathrm{err}}\left(u, v ; t^{N}\right) \geq & W\left(t^{N}\right) e\left(t^{N}\right)+\int_{0}^{t^{N}} w_{\epsilon_{t}}\left(t^{N}-t\right) e(t) d t \\
& -W\left(t^{N}\right) e(0)-\int_{0}^{t^{N}} w_{\epsilon_{t}}(t) e(t) d t \\
& -4 W\left(t^{N}\right)\left\{\left\|f^{\prime}(v)\right\| \epsilon_{t}+\epsilon_{x}\right\}\left|v_{0}\right|_{T V(\mathbb{R})} .
\end{aligned}
$$

Since the functions $w$ and $\eta$ satisfy the properties (2.1), to obtain the above result, we can simply take $\tau=t^{N}$ in Proposition 5.2.

\section{e. The approximation inequality.}

Proposition 7.6 (The approximation inequality). We have

$$
\begin{aligned}
e\left(t^{N}\right) \leq & 2 e(0)+8\left(\epsilon_{x}+\epsilon_{t}\left\|f^{\prime}(v)\right\|\right)\left|v_{0}\right|_{T V(\mathbb{R})} \\
& +2 \lim _{w \rightarrow \chi} \sup _{1 \leq n \leq N}\left\{E_{\text {div }}^{\star}\left(u, v ; t^{n}\right) / W\left(t^{n}\right)\right\} \\
& +2\left\|f^{\prime}(v)\right\|\left|v_{0}\right|_{T V(\mathbb{R})} \Delta t .
\end{aligned}
$$

To prove this result, we need the following auxiliary lemma, which is a slight modification of Lemma 5.4.

Lemma 7.7. Let $\theta:[0, T] \mapsto \mathbb{R}^{+}$be a nonnegative, measurable function such that for all $N \in \mathbb{N}$

$$
W^{\infty}\left(t^{N}\right) \theta\left(t^{N}\right)+\int_{0}^{t^{N}} w_{\epsilon_{t}}^{\infty}\left(t^{N}-t\right) \theta(t) d t \leq W^{\infty}\left(t^{N}\right) C+\int_{0}^{t^{N}} w_{\epsilon_{t}}^{\infty}(t) \theta(t) d t,
$$

where $w^{\infty}=\chi$. Moreover, assume that, for $t \in\left[t^{n}, t^{n+1}\right)$,

$$
\theta(t) \leq \theta\left(t^{n}\right)+c \Delta t
$$

Then, for $\tau \in[0, T]$,

$$
\theta(\tau) \leq 2 C+c \Delta t
$$

Proof. The proof is similar to the proof of Lemma 5.4. If $t^{n} \leq \epsilon_{t}$, we have for $t \in\left[0, t^{n}\right]$ that $w_{\epsilon_{t}}^{\infty}\left(t^{n}-t\right)=w_{\epsilon_{t}}^{\infty}(t) \equiv 1 / 2 \epsilon_{t}$, and so

$$
\theta\left(t^{n}\right) \leq C .
$$

By the second hypothesis on $\theta$, for $t \leq \epsilon_{t}$,

$$
\theta(t) \leq C+c \Delta t
$$

To estimate $\theta(\tau)$ for $\tau \geq \epsilon_{t}$, we proceed as in the proof of Lemma 5.4 to get

$$
\begin{aligned}
\theta(\tau) & \leq C+\left\{\int_{0}^{\epsilon_{t}}\left\{w_{\epsilon_{t}}^{\infty}(t)-w_{\epsilon_{t}}^{\infty}(\tau-t)\right\} d t / W^{\infty}(\tau)\right\}\{C+c \Delta t\} \\
& \leq 2 C+c \Delta t .
\end{aligned}
$$

This completes the proof. 
Proof of Proposition 7.6. This result follows from Proposition 7.5 and Lemma 7.7 with

$$
\begin{aligned}
& \theta\left(t^{n}\right)=e\left(t^{n}\right), \\
& C=4\left\{\left\|f^{\prime}(v)\right\| \epsilon_{t}+\epsilon_{x}\right\}\left|v_{0}\right|_{T V(\mathbb{R})}+\lim _{w \rightarrow \chi} \sup _{1 \leq n \leq N}\left\{E_{\mathrm{div}}^{\star}\left(u, v ; t^{n}\right) / W\left(t^{n}\right)\right\}, \\
& c=\left\|f^{\prime}(v)\right\| \mid v_{0} \|_{T V(\mathbb{R})} .
\end{aligned}
$$

This completes the proof.

f. The estimate of the dual form $E_{\text {div }}^{\star}\left(u, v ; t^{n}\right) / W\left(t^{n}\right)$. The following result is the analog of Proposition 5.5.

Proposition 7.8. We have

$$
\lim _{w \rightarrow \chi} \sup _{1 \leq n \leq N}\left\{E_{\text {div }}^{\star}\left(u, v ; t^{n}\right) / W\left(t^{n}\right)\right\} \leq \frac{2}{\epsilon_{x}} t^{N}\left|v_{0}\right|_{T V(\mathbb{R})}\left\|\nu_{v}\right\|+E_{\text {small }}
$$

where the quantity $\left\|\nu_{v}\right\|$ is defined in Theorem 6.1 and the small term $E_{\text {small }}$ is given by

$$
E_{\text {small }}=c_{1} t^{N}\left|v_{0}\right|_{T V(\mathbb{R})}\left\|\nu_{v}\right\|+c_{2}\left\|f^{\prime}(v)\right\|\left|v_{0}\right|_{T V(\mathbb{R})},
$$

where

$$
\begin{aligned}
& c_{1}=2 \frac{|\eta|_{T V(\mathbb{R})}-1}{\epsilon_{x}}+2 \frac{|\eta|_{T V(\mathbb{R})}}{\epsilon_{x}} \frac{\Delta t}{\epsilon_{t}}, \\
& c_{2}=\left(\frac{\left|\eta^{\prime}\right|_{T V(\mathbb{R})}}{6}\left(2+\frac{\Delta t}{\epsilon_{t}}\right) \frac{(\Delta x)^{2}}{\epsilon_{x}^{2}}+\frac{|\eta|_{T V(\mathbb{R})}}{\epsilon_{t} \epsilon_{x}}(\Delta t)^{2}\left\|f^{\prime}(v)\right\|\right) t^{N}+\left(2+4 \frac{\Delta t}{\epsilon_{t}}\right) \Delta t .
\end{aligned}
$$

We want to point out that it is very simple to show that the dual form $E_{\text {div }}^{\star}\left(u, v ; t^{n}\right) / W\left(t^{n}\right)$ can be bounded by a term proportional to $\Delta x / \epsilon_{x}+\Delta t / \epsilon_{t}$. However, to obtain the finer estimate of Proposition 7.8, an extra effort must be made. We thus proceed in several steps. In what follows, whenever no confusion is possible, we abbreviate $F\left(u\left(t^{n}, x^{\prime}\right), v(t, x)\right)$ and $\left|F\left(u\left(t^{n}, x^{\prime}\right), v(t, x)\right)\right|$ by $F$ and $|F|$, respectively.

First step: Rewriting the dual form $E_{\text {div }}^{\star}(u, v ; t)$. We start by suitably rewriting the dual form. To state our result, we need to define the following regularizations of the function $\varphi$ :

$$
\begin{aligned}
\bar{\varphi}\left(t, x, t^{n+1}, x^{\prime}\right) & =\frac{1}{\Delta t} \int_{t^{n}}^{t^{n+1}} \varphi\left(t, x, s, x^{\prime}\right) d s \\
\phi\left(t, x, t^{n+1}, x^{\prime}\right) & =\frac{1}{\Delta x} \int_{-\Delta x / 2}^{\Delta x / 2} \varphi\left(t, x+\rho, t^{n+1}, x^{\prime}\right) d \rho \\
\hat{\varphi}\left(t, x, t^{n+1}, x^{\prime}\right) & =\frac{1}{\Delta x} \int_{-\Delta x / 2}^{\Delta x / 2} \phi\left(t, x+\rho, t^{n+1}, x^{\prime}\right) d \rho
\end{aligned}
$$


Lemma 7.9. We have

$$
E_{\mathrm{div}}^{\star}\left(u_{h}, v ; t^{N}\right) \leq T E_{\mathrm{visc}}\left(u, v ; t^{N}\right)+T E_{\mathrm{hot}}\left(u, v ; t^{N}\right)
$$

where

$$
\begin{aligned}
T E_{\mathrm{visc}}\left(u, v ; t^{N}\right)= & +\frac{1}{2} \sum_{n=0}^{N-1} \int_{\mathbb{R}} \int_{0}^{t^{N}} \int_{\mathbb{R}}\left\{\Delta t F \hat{\varphi}_{x t}+\Delta x|F| \hat{\varphi}_{x x}\right\} d x d t d x^{\prime} \Delta t \\
& -\frac{1}{2} \sum_{n=0}^{N-1} \int_{\mathbb{R}} \int_{\mathbb{R}}\left\{\Delta t F\left(u\left(t^{n}, x^{\prime}\right), v\left(t^{N}, x\right)\right)\right. \\
\left.\cdot \hat{\varphi}_{x}\left(t^{N}, x, t^{n+1}, x^{\prime}\right)\right\} d x d x^{\prime} \Delta t & \\
& +\frac{1}{2} \sum_{n=0}^{N-1} \int_{\mathbb{R}} \int_{\mathbb{R}}\left\{\Delta t F\left(u\left(t^{n}, x^{\prime}\right), v(0, x)\right)\right. \\
T E_{\mathrm{hot}}\left(u, v ; t^{N}\right)= & -\sum_{n=0}^{N-1} \int_{\mathbb{R}} \int_{0}^{t^{N}} \int_{\mathbb{R}} F \Phi d x d t d x^{\prime} \Delta t \\
& +\frac{1}{2} \sum_{n=0}^{N-1} \int_{\mathbb{R}} \int_{\mathbb{R}} \Delta t F\left(u\left(t^{n}, x^{\prime}\right), v\left(t^{N}, x\right)\right) \\
& \left.-\frac{1}{2} \sum_{n=0}^{N-1} \int_{\mathbb{R}} \int_{\mathbb{R}} \Delta t F\left(u\left(t^{n}, x^{\prime}\right), v(0, x)\right)\right\} d x d x^{\prime} \Delta t,
\end{aligned}
$$

and

$$
\begin{aligned}
\Phi=\{ & \left.\frac{1}{2}\left(\phi_{x}\left(t, x+\Delta x / 2, t^{n+1}, x^{\prime}\right)+\phi_{x}\left(t, x-\Delta x / 2, t^{n+1}, x^{\prime}\right)\right)-\bar{\varphi}_{x}\left(t, x, t^{n+1}, x^{\prime}\right)\right\} \\
& +\frac{\Delta t}{2} \hat{\varphi}_{x t}\left(t, x, t^{n+1}, x^{\prime}\right) .
\end{aligned}
$$

The fact which suggested the above decomposition of the dual form is that the term $T E_{\mathrm{visc}}\left(u, v ; t^{N}\right)$ contains the information of the main term of the local truncation error of the numerical scheme, that is, it contains the information about the effective viscosity of the scheme. To see this, assume that $U(w)=|w|$ and that $v$ is smooth. After a couple of integration by parts, the term $T E_{\mathrm{visc}}\left(u, v ; t^{N}\right)$ can be written as

$$
\frac{1}{2} \sum_{n=0}^{N-1} \int_{\mathbb{R}} \int_{0}^{t^{N}} \int_{\mathbb{R}}\left\{\Delta t F_{t}+\Delta x|F|_{x}\right\} \hat{\varphi}_{x} d x d t d x^{\prime} \Delta t
$$

and since

$$
\begin{aligned}
\frac{1}{2}\left\{\Delta t F(u, v)_{t}+\Delta x|F(u, v)|_{x}\right\} & =-U^{\prime}(u, v) \frac{1}{2}\left\{\Delta t f^{\prime}(v) v_{t}+\Delta x\left|f^{\prime}(v)\right| v_{x}\right\} \\
& =-U^{\prime}(u, v) \frac{\Delta x}{2}\left\{-\Delta t\left(f^{\prime}(v)\right)^{2}+\Delta x\left|f^{\prime}(v)\right|\right\} v_{x} \\
& =-U^{\prime}(u, v) \nu(v) v_{x}
\end{aligned}
$$


our claim follows. The term $T E_{\text {hot }}\left(u, v ; t^{N}\right)$ contains the high-order terms of the truncation error of the scheme.

Proof of Lemma 7.9. Using the notation introduced above, and using the fact that $v$ is the entropy solution, we have

$$
\begin{aligned}
E_{\mathrm{div}}^{\star}\left(u_{h}, v ; t^{N}\right) \leq & \int_{0}^{t^{N}} \int_{\mathbb{R}} \int_{0}^{t^{N}} \int_{\mathbb{R}} F\left(v(t, x), u\left(t^{\prime}, x^{\prime}\right)\right) \varphi_{x}\left(t, x, t^{\prime}, x^{\prime}\right) d x d t d x^{\prime} d t^{\prime} \\
& -\sum_{n=0}^{N-1} \int_{\mathbb{R}} \int_{0}^{t^{N}} \int_{\mathbb{R}} \tilde{\Phi} F d x d t d x^{\prime} \Delta t \\
& +\frac{1}{2} \sum_{n=0}^{N-1} \int_{\mathbb{R}} \int_{0}^{t^{N}} \int_{\mathbb{R}} \Delta x \hat{\varphi}_{x x}|F| d x d t d x^{\prime} \Delta t
\end{aligned}
$$

where

$$
\tilde{\Phi}=\frac{1}{2}\left(\phi_{x}\left(t, x+\Delta x / 2, t^{n+1}, x^{\prime}\right)+\phi_{x}\left(t, x-\Delta x / 2, t^{n+1}, x^{\prime}\right)\right) .
$$

Taking into account that for $U(w)=|w|$ we have $F(v, u)=F(u, v)$, and writing

$$
\begin{aligned}
\int_{0}^{t^{N}} F\left(v(t, x), u\left(t^{\prime}, x^{\prime}\right)\right) \varphi_{x}\left(t, x, t^{\prime}, x^{\prime}\right) d t^{\prime} & =\sum_{n=0}^{N-1} F \bar{\varphi}_{x}\left(t, x, t^{n+1}, x^{\prime}\right)\left(t^{n+1}-t^{n}\right) \\
& =\sum_{n=0}^{N-1} F \bar{\varphi}_{x}\left(t, x, t^{n+1}, x^{\prime}\right) \Delta t
\end{aligned}
$$

we obtain

$$
\begin{aligned}
E_{\mathrm{div}}^{\star}\left(u_{h}, v ; t^{N}\right) \leq & -\sum_{n=0}^{N-1} \int_{\mathbb{R}} \int_{0}^{t^{N}} \int_{\mathbb{R}} F\left\{\tilde{\Phi}-\bar{\varphi}_{x}\left(t, x, t^{n+1}, x^{\prime}\right)\right\} d x d t d x^{\prime} \Delta t \\
& +\frac{1}{2} \sum_{n=0}^{N-1} \int_{\mathbb{R}} \int_{0}^{t^{N}} \int_{\mathbb{R}} \Delta x|F| \hat{\varphi}_{x x} d x d t d x^{\prime} \Delta t
\end{aligned}
$$

Adding and subtracting the term

$$
\frac{1}{2} \sum_{n=0}^{N-1} \int_{\mathbb{R}} \int_{0}^{t^{N}} \int_{\mathbb{R}} \Delta t F \hat{\varphi}_{x t} d x d t d x^{\prime} \Delta t
$$

we get

$$
\begin{aligned}
E_{\mathrm{div}}^{\star}\left(u_{h}, v ; t^{N}\right) \leq & -\sum_{n=0}^{N-1} \int_{\mathbb{R}} \int_{0}^{t^{N}} \int_{\mathbb{R}} F \Phi d x d t d x^{\prime} \Delta t \\
& +\frac{1}{2} \sum_{n=0}^{N-1} \int_{\mathbb{R}} \int_{0}^{t^{N}} \int_{\mathbb{R}}\left\{\Delta t F \hat{\varphi}_{x t}+\Delta x|F| \hat{\varphi}_{x x}\right\} d x d t d x^{\prime} \Delta t .
\end{aligned}
$$

The result now follows easily by using the definitions of the terms $T E_{\mathrm{visc}}\left(u, v ; t^{N}\right)$ and $T E_{\text {hot }}\left(u, v ; t^{N}\right)$. This completes the proof. 
Second step: The functions $\hat{\varphi}$ and $\Phi$. From Lemma 7.9, it is clear that what we have to do next is to express the functions $\hat{\varphi}$ and $\Phi$ in terms of the function $\varphi$.

Lemma 7.10. We have

$$
\hat{\varphi}\left(t, x, t^{\prime}, x^{\prime}\right)=\frac{1}{\Delta x} \int_{-\Delta x}^{\Delta x}\left(1-\frac{|\rho|}{\Delta x}\right) \varphi\left(t, x, t^{\prime}, x^{\prime}-\rho\right) d \rho,
$$

and

$$
\Phi=\Phi_{x x^{\prime} x^{\prime}}^{1}+\Phi_{x x^{\prime} x^{\prime}}^{2}+\Phi_{t t^{\prime} x^{\prime}}^{3}
$$

where the functions $\Phi^{i}=\Phi^{i}\left(t, x, t^{n+1}, x^{\prime}\right), i=1,2,3$, are given by

$$
\begin{aligned}
\Phi^{1} & =\frac{\Delta x}{6 \Delta t} \int_{0}^{\Delta t} \int_{-\Delta x}^{\Delta x}\left(1-\frac{|\rho|}{\Delta x}\right)^{3} \varphi\left(t, x, t^{n+1}-s, x^{\prime}-\rho\right) d \rho d s \\
\Phi^{2} & =\frac{\Delta x}{6} \int_{-\Delta x}^{\Delta x}\left(1-\frac{|\rho|}{\Delta x}\right)^{2}\left(\frac{1}{2}+\frac{|\rho|}{\Delta x}\right) \varphi\left(t, x, t^{n+1}, x^{\prime}-\rho\right) d \rho, \\
\Phi^{3} & =-\frac{\Delta t}{2 \Delta x} \int_{0}^{\Delta t} \int_{-\Delta x}^{\Delta x}\left(1-\frac{s}{\Delta t}\right)^{2}\left(1-\frac{|\rho|}{\Delta x}\right) \varphi\left(t, x, t^{n+1}-s, x^{\prime}-\rho\right) d \rho d s .
\end{aligned}
$$

Proof. The first equality follows from the definition of $\hat{\varphi},(7.3 \mathrm{c})$. To obtain the expression of $\Phi$, we first rewrite $\Phi$ as

$$
\Phi=\tilde{\Phi}^{1}+\tilde{\Phi}^{2}+\tilde{\Phi}^{3}
$$

where

$$
\begin{aligned}
& \tilde{\Phi}^{1}=\overline{\hat{\varphi}}_{x}\left(t, x, t^{n+1}, x^{\prime}\right)-\bar{\varphi}_{x}\left(t, x, t^{n+1}, x^{\prime}\right), \\
& \tilde{\Phi}^{2}=\frac{1}{2}\left(\phi_{x}\left(t, x+\Delta x / 2, t^{n+1}, x^{\prime}\right)+\phi_{x}\left(t, x-\Delta x / 2, t^{n+1}, x^{\prime}\right)\right)-\hat{\varphi}_{x}\left(t, x, t^{n+1}, x^{\prime}\right) \\
& \tilde{\Phi}^{3}=\hat{\varphi}_{x}\left(t, x, t^{n+1}, x^{\prime}\right)-\overline{\hat{\varphi}}_{x}\left(t, x, t^{n+1}, x^{\prime}\right)-\frac{\Delta t}{2} \hat{\varphi}_{t x}\left(t, x, t^{n+1}, x^{\prime}\right) .
\end{aligned}
$$

To show that $\tilde{\Phi}^{1}=\Phi_{x x^{\prime} x^{\prime}}^{1}, \tilde{\Phi}^{2}=\Phi_{x x^{\prime} x^{\prime}}^{2}, \tilde{\Phi}^{3}=\Phi_{t t^{\prime} x^{\prime}}^{3}$, we use the definitions of $\phi, \hat{\varphi}$ and $\bar{\varphi}$ given by (7.3), and we perfom several simple integration by parts.

Third step: Estimating $T E_{\text {visc }}\left(u, v ; t^{N}\right)$. We are now ready to estimate the term $T E_{\text {visc }}\left(u, v ; t^{N}\right)$.

Lemma 7.11. We have

$$
T E_{\mathrm{visc}}\left(u, v ; t^{N}\right) \leq T_{0} t^{N}\left|v_{0}\right|_{T V(\mathbb{R})}\left\|\nu_{v}\right\|,
$$

where

$$
T_{0}=\sup _{\substack{t \in\left(0, t^{N}\right) \\ x \in \mathbb{R}}}\left\{\sum_{n=0}^{N-1} \int_{\mathbb{R}}\left|\hat{\varphi}_{x}\left(t, x, t^{n+1}, x^{\prime}\right)\right| d x^{\prime} \Delta t\right\} .
$$


In the proof of the above result we denote by $[G]$ the jump of the function $G(v)$ at the point $(x(t), t)$, that is, the value $G(v(t, x(t)+0))-G(v(t, x(t)-0)) \equiv$ $G\left(v^{+}\right)-G\left(v^{-}\right)$. Also, for the sake of simplicity, we do not render explicit the dependence of $F$ and $|F|$ on $u$; recall that

$$
\begin{aligned}
& F(u, v)=\int_{v}^{u} f^{\prime}(s) U^{\prime}(s-v) d s \\
& |F|(u, v)=\int_{v}^{u}\left|f^{\prime}(s)\right| U^{\prime}(s-v) d s .
\end{aligned}
$$

Proof of Lemma 7.11. It is enough to prove the result in the case in which the entropy solution $v$ is smooth everywhere except on a single curve $\mathcal{C}=\{(x(t), t)$ : $\left.t \in\left(0, t^{N}\right)\right\}$ since the general case follows easily from this case, as we have shown in the proof of Proposition 5.5.

After a couple of simple integration by parts, we can rewrite $T E_{\mathrm{visc}}\left(u, v ; t^{N}\right)$ as follows:

$$
T E_{\mathrm{visc}}\left(u, v ; t^{N}\right)=\sum_{n=0}^{N-1} \int_{\mathbb{R}} \Psi\left(t^{n+1}, x^{\prime}\right) d x^{\prime} \Delta t
$$

where

$$
\begin{aligned}
\Psi\left(t^{n+1}, x^{\prime}\right)= & -\frac{1}{2} \int_{0}^{t^{N}} \int_{-\infty}^{x(t)}\left\{\Delta t F_{t}+\Delta x|F|_{x}\right\} \hat{\varphi}_{x} d x d t \\
& -\frac{1}{2} \int_{0}^{t^{N}} \int_{x(t)}^{\infty}\left\{\Delta t F_{t}+\Delta x|F|_{x}\right\} \hat{\varphi}_{x} d x d t \\
& -\frac{1}{2} \int_{0}^{t^{N}}\left\{-\Delta t[F] \frac{[f]}{[v]}+\Delta x[|F|]\right\} \hat{\varphi}_{x} d t
\end{aligned}
$$

Setting

$$
\tilde{\nu}\left(v^{-}, v^{+} ; u\right)=\frac{\Delta x}{2}\left\{\frac{[|F|]}{[v]}-\frac{\Delta t}{\Delta x} \frac{[F]}{[v]} \frac{[f]}{[v]}\right\}
$$

and writing $\tilde{\nu}$ instead of $\tilde{\nu}\left(v^{-}, v^{+} ; u\left(t^{n}, x^{\prime}\right)\right)$, we have

$$
\Psi\left(t^{n+1}, x^{\prime}\right)=-\int_{0}^{t^{N}}\left\{\int_{-\infty}^{x(t)} \tilde{\nu} v_{x} \hat{\varphi}_{x} d x+\tilde{\nu}[v] \hat{\varphi}_{x}+\int_{x(t)}^{\infty} \tilde{\nu} v_{x} \hat{\varphi}_{x} d x\right\} d t
$$

Setting

$$
\|\tilde{\nu}\|=\sup _{\substack{t \in\left(0, t^{N}\right) \\ x \in \mathbb{R}}} \sup _{u \in \mathbb{R}}|\tilde{\nu}(v(t, x-0), v(t, x+0) ; u)|,
$$

we obtain, after taking absolute values,

$\left|\Psi\left(t^{n+1}, x^{\prime}\right)\right| \leq\|\tilde{\nu}\| \int_{0}^{t^{N}}\left\{\int_{-\infty}^{x(t)}\left|v_{x}\right|\left|\hat{\varphi}_{x}\right| d x+|[v]|\left|\hat{\varphi}_{x}\right|+\int_{x(t)}^{\infty}\left|v_{x}\right|\left|\hat{\varphi}_{x}\right| d x\right\} d t$. 
Inserting this upper bound into the right-hand side of the definition of $T E_{\text {visc }}\left(u, v ; t^{N}\right)$, we get

$$
\begin{aligned}
T E_{\mathrm{visc}}\left(u, v ; t^{N}\right) & \leq T_{0}\|\tilde{\nu}\| \int_{0}^{t^{N}}\left\{\int_{-\infty}^{x(t)}\left|v_{x}\right| d x+|[v]|+\int_{x(t)}^{\infty}\left|v_{x}\right| d x\right\} d t \\
& \leq T_{0} t^{N}|v|_{L^{\infty}\left(0, t^{N} ; T V(\mathbb{R})\right)}\|\tilde{\nu}\| .
\end{aligned}
$$

To prove the result, it remains to show that $\left|\tilde{\nu}\left(v^{-}, v^{+} ; u\right)\right| \leq \nu\left(v^{-}, v^{+}\right)$. To do this, set $N(u)=\tilde{\nu}\left(v^{-}, v^{+} ; u\right)$ and note that

$\partial_{u} N(u)=\frac{\Delta x}{2} \frac{U^{\prime}\left(u-v^{+}\right)-U^{\prime}\left(u-v^{-}\right)}{v^{+}-v^{-}}\left\{\left|f^{\prime}(u)\right|-\frac{\Delta t}{\Delta x}\left(\frac{f\left(v^{+}\right)-f\left(v^{-}\right)}{v^{+}-v^{-}}\right) f^{\prime}(u)\right\}$.

This implies that $N(u)$ is constant when $u$ does not lie between $v^{-}$and $v^{+}$and that, since the stability condition (6.2) is satisfied, $N$ is monotone. Hence,

$$
|N(u)| \leq \max \left\{N\left(v^{+}\right), N\left(v^{-}\right)\right\}=\nu\left(v^{-}, v^{+}\right) .
$$

This completes the proof.

The term $T_{0}$ will be estimated in the fifth step below.

Fourth step: Estimating $T E_{\text {hot }}\left(u, v ; t^{N}\right)$. We are now ready to estimate the term $T E_{\mathrm{hot}}\left(u, v ; t^{N}\right)$. We start with the following result.

Lemma 7.12. We have

$$
T E_{\mathrm{hot}}\left(u, v ; t^{N}\right) \leq c\left\|f^{\prime}(v)\right\|\left|v_{0}\right|_{T V(\mathbb{R})},
$$

where $c=\left(T_{1}+T_{2}+\left\|f^{\prime}(v)\right\| T_{3}\right) t^{N}+2\left(T_{4}+T_{5}\right)$, and

$$
\begin{aligned}
& T_{1}=\sup _{\substack{t \in\left(0, t^{N}\right) \\
x \in \mathbb{R}}}\left\{\sum_{n=0}^{N-1} \int_{\mathbb{R}}\left|\Phi_{x^{\prime} x^{\prime}}^{1}\left(t, x, t^{n+1}, x^{\prime}\right)\right| d x^{\prime} \Delta t\right\}, \\
& T_{2}=\sup _{\substack{t \in\left(0, t^{N}\right) \\
x \in \mathbb{R}}}\left\{\sum_{n=0}^{N-1} \int_{\mathbb{R}}\left|\Phi_{x^{\prime} x^{\prime}}^{2}\left(t, x, t^{n+1}, x^{\prime}\right)\right| d x^{\prime} \Delta t\right\}, \\
& T_{3}=\sup _{\substack{t \in\left(0, t^{N}\right) \\
x \in \mathbb{R}}}\left\{\sum_{n=0}^{N-1} \int_{\mathbb{R}}\left|\Phi_{t^{\prime} x^{\prime}}^{3}\left(t, x, t^{n+1}, x^{\prime}\right)\right| d x^{\prime} \Delta t\right\}, \\
& T_{4}=\sup _{\substack{t \in\left\{0, t^{N}\right\} \\
x \in \mathbb{R}}}\left\{\sum_{n=0}^{N-1} \int_{\mathbb{R}}\left|\Phi_{t^{\prime}}^{3}\left(t, x, t^{n+1}, x^{\prime}\right)\right| d x^{\prime} \Delta t\right\}, \\
& T_{5}=\sup _{\substack{t \in\left\{0, t^{N}\right\} \\
x \in \mathbb{R}}}\left\{\sum_{n=0}^{N-1} \int_{\mathbb{R}} \frac{1}{2} \Delta t\left|\hat{\varphi}\left(t, x, t^{n+1}, x^{\prime}\right)\right| d x^{\prime} \Delta t\right\} .
\end{aligned}
$$

Proof. First, assume that the entropy solution $v$ is smooth. By using Lemma 7.10, we rewrite $T E_{\text {hot }}\left(u, v ; t^{N}\right)$ as follows: 


$$
\begin{aligned}
T E_{\mathrm{hot}}\left(u, v ; t^{N}\right)= & -\sum_{n=0}^{N-1} \int_{\mathbb{R}} \int_{0}^{t^{N}} \int_{\mathbb{R}} F\left\{\Phi_{x x^{\prime} x^{\prime}}^{1}+\Phi_{x x^{\prime} x^{\prime}}^{2}+\Phi_{t t^{\prime} x^{\prime}}^{3}\right\} d x d t d x^{\prime} \Delta t \\
& +\frac{1}{2} \sum_{n=0}^{N-1} \int_{\mathbb{R}} \int_{\mathbb{R}} \Delta t F\left(u\left(t^{n}, x^{\prime}\right), v\left(t^{N}, x\right)\right) \hat{\varphi}_{x}\left(t^{N}, x, t^{n+1}, x^{\prime}\right) d x d x^{\prime} \Delta t \\
& -\frac{1}{2} \sum_{n=0}^{N-1} \int_{\mathbb{R}} \int_{\mathbb{R}} \Delta t F\left(u\left(t^{n}, x^{\prime}\right), v(0, x)\right) \hat{\varphi}_{x}\left(0, x, t^{n+1}, x^{\prime}\right) d x d x^{\prime} \Delta t .
\end{aligned}
$$

Integrating by parts, we get

$$
\begin{aligned}
T E_{\mathrm{hot}}\left(u, v ; t^{N}\right)= & \sum_{n=0}^{N-1} \int_{\mathbb{R}} \int_{0}^{t^{N}} \int_{\mathbb{R}} F_{x}\left\{\Phi_{x^{\prime} x^{\prime}}^{1}+\Phi_{x^{\prime} x^{\prime}}^{2}\right\} d x d t d x^{\prime} \Delta t \\
& +\sum_{n=0}^{N-1} \int_{\mathbb{R}} \int_{0}^{t^{N}} \int_{\mathbb{R}} F_{t} \Phi_{t^{\prime} x^{\prime}}^{3} d x d t d x^{\prime} \Delta t \\
& \left.-\sum_{n=0}^{N-1} \int_{\mathbb{R}} \int_{\mathbb{R}} F\left(u\left(t^{n}, x^{\prime}\right), v\left(t^{N}, x\right)\right) \Phi_{t^{\prime} x^{\prime}}^{3}\left(t^{N}, x, t^{n+1}, x^{\prime}\right)\right\} d x d x^{\prime} \Delta t \\
& \left.+\sum_{n=0}^{N-1} \int_{\mathbb{R}} \int_{\mathbb{R}} F\left(u\left(t^{n}, x^{\prime}\right), v(0, x)\right) \Phi_{t^{\prime} x^{\prime}}^{3}\left(0, x, t^{n+1}, x^{\prime}\right)\right\} d x d x^{\prime} \Delta t \\
& -\frac{1}{2} \sum_{n=0}^{N-1} \int_{\mathbb{R}} \int_{\mathbb{R}} F_{x}\left(u\left(t^{n}, x^{\prime}\right), v\left(t^{N}, x\right)\right) \Delta t \hat{\varphi}\left(t^{N}, x, t^{n+1}, x^{\prime}\right) d x d x^{\prime} \Delta t \\
& +\frac{1}{2} \sum_{n=0}^{N-1} \int_{\mathbb{R}} \int_{\mathbb{R}} F_{x}\left(u\left(t^{n}, x^{\prime}\right), v(0, x)\right) \Delta t \hat{\varphi}\left(0, x, t^{n+1}, x^{\prime}\right) d x d x^{\prime} \Delta t .
\end{aligned}
$$

Taking into account that $\Phi_{t^{\prime} x^{\prime}}^{3}=-\Phi_{t^{\prime} x}^{3}$, we use integration by parts on the third and fourth terms of the right-hand side. We then take absolute values and use the inequalities

$$
\begin{aligned}
& \left|F_{x}\right| \leq\left|f^{\prime}(v)\right|\left|v_{x}\right|, \\
& \left|F_{t}\right| \leq\left|f^{\prime}(v)\right|^{2}\left|v_{x}\right|,
\end{aligned}
$$

to get

$$
\begin{aligned}
T E_{\mathrm{hot}}\left(u, v ; t^{N}\right) \leq & \left\{T_{1}+T_{2}\right\} \int_{0}^{t^{N}} \int_{\mathbb{R}}\left|f^{\prime}(v)\right|\left|v_{x}\right| d x d t+T_{3} \int_{0}^{t^{N}} \int_{\mathbb{R}}\left|f^{\prime}(v)\right|^{2}\left|v_{x}\right| d x d t \\
& +T_{4} \int_{\mathbb{R}}\left|f^{\prime}\left(v\left(t^{N}\right)\right)\right|\left|v_{x}\left(t^{N}\right)\right| d x+T_{4} \int_{\mathbb{R}}\left|f^{\prime}\left(v_{0}\right)\right|\left|v_{0 x}\right| d x \\
& +T_{5} \int_{\mathbb{R}}\left|f^{\prime}\left(v\left(t^{N}\right)\right)\right|\left|v_{x}\left(t^{N}\right)\right| d x+T_{5} \int_{\mathbb{R}}\left|f^{\prime}\left(v_{0}\right)\right|\left|v_{0 x}\right| d x
\end{aligned}
$$

and the inequality follows. The result for general $v$ follows from a classical density argument. This completes the proof. 
Fifth step: Estimates of the terms $T_{i}, i=0, \ldots, 5$.

Lemma 7.13. If we let $w$ tend to $\chi$, we get

$$
\begin{aligned}
& T_{0} \leq\left(1+\frac{\Delta t}{\epsilon_{t}}\right) \frac{2|\eta|_{T V(\mathbb{R})}}{\epsilon_{x}} W\left(t^{N}\right) \\
& T_{1} \leq \frac{(\Delta x)^{2}\left|\eta^{\prime}\right|_{T V(\mathbb{R})}}{6 \epsilon_{x}^{2}} W\left(t^{N}\right) \\
& T_{2} \leq \frac{(\Delta x)^{2}\left|\eta^{\prime}\right|_{T V(\mathbb{R})}}{6 \epsilon_{x}^{2}}\left(1+\frac{\Delta t}{\epsilon_{t}}\right) W\left(t^{N}\right), \\
& T_{3} \leq \frac{|\eta|_{T V(\mathbb{R})}}{\epsilon_{t} \epsilon_{x}}(\Delta t)^{2} W\left(t^{N}\right) \\
& T_{4} \leq \frac{(\Delta t)^{2}}{\epsilon_{t}} W\left(t^{N}\right) \\
& T_{5} \leq \Delta t\left(1+\frac{\Delta t}{\epsilon_{t}}\right) W\left(t^{N}\right)
\end{aligned}
$$

Proof. All the terms $T_{i}$ are of the form $\sup _{\substack{t \in\left(0, t^{N}\right) \\ x \in \mathbb{R}}} \Theta_{i}$. In what follows, we only work with $\Theta_{i}$. Throughout the proof, we use the fact that

$$
\lim _{w \rightarrow \chi} \sup _{t \in\left(0, t^{N}\right)} \frac{\left|w_{\epsilon_{t}}\right| T V\left(-t, t^{N}-t\right)}{\int_{0}^{t^{n}} w_{\epsilon_{t}}\left(t^{\prime}\right) d t^{\prime}} \leq \frac{2}{\epsilon_{t}},
$$

which can easily be established by direct calculation.

Let us start with $\Theta_{0}$. By definition,

$$
\Theta_{0}=\sum_{n=0}^{N-1} \int_{\mathbb{R}}\left|\hat{\varphi}_{x}\left(t, x, t^{n+1}, x^{\prime}\right)\right| d x^{\prime} \Delta t .
$$

By using the definitions of $\varphi$ and $\hat{\varphi}$, as well as Lemma 7.10, we can rewrite $\Theta_{0}$ as follows:

$$
\begin{aligned}
\Theta_{0}= & \left\{\sum_{n=0}^{N-1} w_{\epsilon_{t}}\left(t-t^{n+1}\right) \Delta t\right\} \\
& \cdot\left\{\frac{1}{\Delta x} \int_{-\Delta x}^{\Delta x}\left(1-\frac{|\rho|}{\Delta x}\right) \int_{\mathbb{R}}\left|\left(\eta_{\epsilon_{x}}\left(x-x^{\prime}+\rho\right)\right)_{x^{\prime}}\right| d x^{\prime} d \rho\right\},
\end{aligned}
$$

and so,

$$
\begin{aligned}
& \Theta_{0} \leq\left\{\int_{0}^{t^{N}} w_{\epsilon_{t}}\left(t-t^{\prime}\right) d t^{\prime}-\sum_{n=0}^{N-1} \int_{t^{n}}^{t^{n+1}}\left(s-t^{n}\right) w_{\epsilon_{t}}^{\prime}(t-s) d s\right\} \\
& \cdot\left\{\frac{1}{\Delta x} \int_{-\Delta x}^{\Delta x}\left(1-\frac{|\rho|}{\Delta x}\right) d \rho\right\} \frac{|\eta|_{T V(\mathbb{R})}}{\epsilon_{x}} \\
&=\left\{2 W\left(t^{n}\right)+\Delta t\left|w_{\epsilon_{t}}\right|_{T V\left(-t, t^{n}-t\right)}\right\} \frac{|\eta|_{T V(\mathbb{R})}}{\epsilon_{x}} .
\end{aligned}
$$


Relation (7.4) leads to

$$
\Theta_{0} \leq 2\left(1+\frac{\Delta t}{\epsilon_{t}}\right) W\left(t^{N}\right) \frac{|\eta|_{T V(\mathbb{R})}}{\epsilon_{x}}
$$

which proves the estimate of $T_{0}$.

Let us prove the second estimate. By definition of $\Theta_{1}$,

$$
\begin{aligned}
\Theta_{1} & =\sum_{n=0}^{N-1} \int_{\mathbb{R}}\left|\Phi_{x^{\prime} x^{\prime}}^{1}\left(t, x, t^{n+1}, x^{\prime}\right)\right| d x^{\prime} \Delta t \\
& =\left\{\int_{0}^{t^{N}} w_{\epsilon_{t}}\left(t-t^{\prime}\right) d t^{\prime}\right\}\left\{\frac{\Delta x}{6} \int_{-\Delta x}^{\Delta x}\left(1-\frac{|\rho|}{\Delta x}\right)^{3} \int_{\mathbb{R}}\left(\eta_{\epsilon_{x}}\left(x-x^{\prime}+\rho\right)_{x^{\prime} x^{\prime}} d x^{\prime} d \rho\right\}\right. \\
& \leq 2 W\left(t^{N}\right)\left\{\frac{\Delta x}{6} \int_{-\Delta x}^{\Delta x}\left(1-\frac{|\rho|}{\Delta x}\right)^{3} \frac{\left|\eta^{\prime}\right| T V(\mathbb{R})}{\epsilon_{x}^{2}} d \rho\right\} \\
& =2 W\left(t^{N}\right) \frac{(\Delta x)^{2}\left|\eta^{\prime}\right|_{T V(\mathbb{R})}}{12 \epsilon_{x}^{2}},
\end{aligned}
$$

and the second estimate follows. The other terms can be analyzed in a similar way.

Sixth step: Proof of Proposition 7.8. From Lemmas 7.11 and 7.13, we have

$$
T E_{\mathrm{visc}}\left(u, v ; t^{N}\right) / W\left(t^{N}\right) \leq \frac{2|\eta|_{T V(\mathbb{R})}}{\epsilon_{x}} t^{N}\left|v_{0}\right|_{T V(\mathbb{R})}\left(1+\frac{\Delta t}{\epsilon_{t}}\right)\left\|\nu_{v}\right\|,
$$

and from Lemmas 7.12 and 7.13, we get

$$
T E_{\mathrm{hot}}\left(u, v ; t^{N}\right) / W\left(t^{N}\right) \leq c\left\|f^{\prime}(v)\right\|\left|v_{0}\right|_{T V(\mathbb{R})},
$$

where

$$
c=\left(\frac{\left|\eta^{\prime}\right|_{T V(\mathbb{R})}}{6}\left(2+\frac{\Delta t}{\epsilon_{t}}\right) \frac{(\Delta x)^{2}}{\epsilon_{x}^{2}}+\frac{|\eta|_{T V(\mathbb{R})}}{\epsilon_{t} \epsilon_{x}}(\Delta t)^{2}\left\|f^{\prime}(v)\right\|\right) t^{N}+\left(2+4 \frac{\Delta t}{\epsilon_{t}}\right) \Delta t .
$$

By Lemma 7.9, Proposition 7.8 follows from the two above inequalities after a simple rearrangement of terms. This completes the proof of Proposition 7.8.

g. Proof of the error estimate. By Propositions 7.6 and 7.8, we have

$$
\begin{aligned}
e\left(t^{N}\right) \leq & 2 e(0)+8\left(\epsilon_{x}+\epsilon_{t}\left\|f^{\prime}(v)\right\|\right)\left|v_{0}\right|_{T V(\mathbb{R})}+\frac{4}{\epsilon_{x}} t^{N}\left|v_{0}\right|_{T V(\mathbb{R})}\left\|\nu_{v}\right\| \\
& +2 E_{\text {small }}+2\left\|f^{\prime}(v)\right\|\left|v_{0}\right|_{T V(\mathbb{R})} \Delta t
\end{aligned}
$$

where $E_{\text {small }}=E_{\text {small }}\left(\eta, \epsilon_{t}, \epsilon_{x}\right)$ is defined in Proposition 7.8.

In the continuous case, both auxiliary functions $w$ and $\eta$ were taken to converge to the function $\chi$. Accordingly, we let $\eta$ go to the function $\chi_{\epsilon}$. We thus use (7.2) to obtain the following inequality:

$$
e\left(t^{N}\right) \leq 2 e(0)+8 \epsilon_{x}\left|v_{0}\right|_{T V(\mathbb{R})}+\frac{4}{\epsilon_{x}} t^{N}\left|v_{0}\right|_{T V(\mathbb{R})}\left\|\nu_{v}\right\|+\Phi\left(\epsilon, \epsilon_{t}, \epsilon_{x}\right),
$$


where

$$
\Phi\left(\epsilon, \epsilon_{t}, \epsilon_{x}\right)=8 \epsilon_{t}\left\|f^{\prime}(v)\right\|\left|v_{0}\right|_{T V(\mathbb{R})}+2 E_{\text {small }}\left(\chi_{\epsilon}, \epsilon_{t}, \epsilon_{x}\right)+2\left\|f^{\prime}(v)\right\|\left|v_{0}\right|_{T V(\mathbb{R})} \Delta t .
$$

Proceeding as in the continuous case, and assuming that $\left\|\nu_{v}\right\| \neq 0$, we take the optimal value $\epsilon_{x}=\sqrt{t^{N}\left\|\nu_{v}\right\| / 2}$ to get

$$
e\left(t^{N}\right) \leq 2 e(0)+4\left|v_{0}\right|_{T V(\mathbb{R})}\left\{8 t^{N}\left\|\nu_{v}\right\|\right\}^{1 / 2}+\Phi\left(\epsilon, \epsilon_{t}, \sqrt{t^{N}\left\|\nu_{v}\right\| / 2}\right) .
$$

Now, we only have to suitably choose the parameters $\epsilon$ and $\epsilon_{t}$. Note that, unlike the continuous case, we cannot let $\epsilon_{t}$ go to zero! By using (7.2), we rewrite $\Phi$ as follows:

$$
\begin{aligned}
\Phi\left(\epsilon, \tilde{\epsilon}_{t} /\left\|f^{\prime}(v)\right\|, \sqrt{t^{N}\left\|\nu_{v}\right\| / 2}\right)= & \left(a_{1} \Delta x+a_{2}(\Delta x)^{1 / 2} \epsilon+a_{3} \tilde{\epsilon}_{t}+a_{4} \Delta x^{3 / 2} \frac{\epsilon}{\tilde{\epsilon}_{t}}\right. \\
& \left.+a_{5} \frac{\Delta x}{\epsilon}+a_{6} \frac{(\Delta x)^{3 / 2}}{\tilde{\epsilon}_{t}}+a_{7} \frac{(\Delta x)^{2}}{\epsilon \tilde{\epsilon}_{t}}\right)\left|v_{0}\right|_{T V(\mathbb{R})},
\end{aligned}
$$

where

$$
\begin{aligned}
& a_{1}=6 \mathrm{cfl}+\frac{8}{3} \zeta \\
& a_{2}=4 \sqrt{2} \kappa+\frac{4}{3} \zeta(\Delta x)^{1 / 2}, \\
& a_{3}=8 \\
& a_{4}=4 \sqrt{2} \mathrm{cfl} \kappa+\frac{2}{3} \zeta \mathrm{cfl}(\Delta x)^{1 / 2}+2 \sqrt{2} \mathrm{cfl}^{2} \zeta \kappa, \\
& a_{5}=\frac{4}{3} \zeta \\
& a_{6}=4 \sqrt{2} \mathrm{cfl} \kappa+2 \sqrt{2} \mathrm{cfl}^{2} \zeta \kappa+8 \mathrm{cfl}^{2}(\Delta x)^{1 / 2}+\frac{4}{3} \mathrm{cfl} \zeta(\Delta x)^{1 / 2} \\
& a_{7}=\frac{2}{3} \zeta \mathrm{cfl},
\end{aligned}
$$

and cfl, $\zeta$, and $\kappa$ are as defined in Theorem 6.1. We can see that if we take $\epsilon=\sqrt{a_{5} / a_{2}}(\Delta x)^{1 / 4}$ and $\tilde{\epsilon}_{t}=\sqrt{a_{6} / a_{3}}(\Delta x)^{3 / 4}$, we have

$$
\Phi\left(\epsilon, \tilde{\epsilon}_{t} /\left\|f^{\prime}(v)\right\|, \sqrt{t^{N}\left\|\nu_{v}\right\| / 2}\right)=\left(b_{1}(\Delta x)^{3 / 4}+b_{2} \Delta x\right)\left|v_{0}\right|_{T V(\mathbb{R})},
$$

where

$$
b_{1}=2\left(\sqrt{a_{2} a_{5}}+\sqrt{a_{3} a_{6}}\right), \quad b_{2}=a_{1}+a_{4} \sqrt{\frac{a_{3} a_{5}}{a_{2} a_{6}}}+a_{7} \sqrt{\frac{a_{2} a_{3}}{a_{5} a_{6}}} .
$$

This proves Theorem 6.1.

Let us finish this section by comparing the size of the parameters $\epsilon_{x}$ and $\epsilon_{t}$ in both of the cases treated in this paper. In the model case treated in $\S 5$, we took $\epsilon_{x}$ proportional to $\left\|\nu_{v}\right\|^{1 / 2}$ and we let $\epsilon_{t}$ go to zero. This gave an indication that in the discrete case treated in this section, it would be reasonable to expect the parameter $\epsilon_{t}$ to go to zero faster than $\epsilon_{x}$. This was indeed the case, since $\epsilon_{x}$ was taken to be proportional to $\left\|\nu_{v}\right\|^{1 / 2}$, which is proportional to $(\Delta x)^{1 / 2}$, while $\epsilon_{t}$ was taken to be proportional to $(\Delta x)^{3 / 4}$. Kuznetsov [15] took both $\epsilon_{x}$ and $\epsilon_{t}$ to be proportional to $(\Delta x)^{1 / 2}$. 


\section{Concluding Remarks}

In this paper, we have showed how to suitably modify the original Kuznetsov approximation theory for scalar conservation laws [15] to obtain a general theory for a priori error estimates. As a first application of this theory, we have obtained a (new) optimal error estimate between the approximate solution defined by the Engquist-Osher scheme in uniform grids and the entropy solution without using any regularity properties of the approximate solution. The only properties explicitly used are that the Engquist-Osher scheme satisfies a (i) local entropy inequality, that (ii) has conservation form, and that (iii) has a two-point splitting entropy flux.

The results of this paper will be extended in several directions. In a forthcoming paper, we obtain optimal error estimates for numerical schemes that do not possess splitting fluxes, like Godunov and Glimm schemes. In another paper, we consider multidimensional schemes satisfying the three properties mentioned above. The fact that we are not forced to obtain regularity properties of the approximate solution allows us to obtain optimal error estimates for these (necessarily monotone) schemes defined in general triangulations.

That it is possible 'to pass to the limit' (and to actually obtain not just convergence but error estimates) without using any regularity properties of the approximate solution might sound strange. After all, a great deal of effort has been invested into devising and refining methods, like the method of compensated compactness, that allow to 'pass to the limit' with the weakest possible regularity of the functions of the sequence under consideration. However, we must point out that we are strongly using the fact that we already know the existence, uniqueness, and regularity properties of the solution to which we want to converge. In this paper, we have shown how to use the regularity properties of the exact solution instead of the regularity properties of the approximate solution to get the error estimates. How to apply this idea to hyperbolic systems constitutes an exciting challenge.

\section{REFERENCES}

1. B. Cockburn, Quasimonotone schemes for scalar conservation laws, I, SIAM J. Numer. Anal. 26 (1989), 1325-1341. MR 91b:65106

2. _ Quasimonotone schemes for scalar conservation laws, II, SIAM J. Numer. Anal. 27 (1990), 247-258. MR 91b:65107

3. __ Quasimonotone schemes for scalar conservation laws, III, SIAM J. Numer. Anal. 27 (1990), 259-276. MR 91b:65107

4. B. Cockburn, F. Coquel, and P. LeFloch, Convergence of the finite volume method for multidimensional conservation laws, SIAM J. Numer. Anal. 32 (1995), 687-705.

5. _ An error estimate for finite volume methods for conservations laws, Math. Comp. 64 (1994), 77-103. MR 95d:65078

6. B. Cockburn and H. Gau, A posteriori error estimates for general numerical schemes for conservations laws, Mat. Apl. Comput. 14 (1995), 37-47.

7. B. Cockburn and P.-A. Gremaud, An error estimate for finite element methods for conservations laws, University of Minnesota Supercomputer Institute Research Report 93-128, SIAM J. Numer. Anal. (to appear).

8. F. Coquel and Ph. Le Floch, Convergence of finite difference schemes for conservation laws in several space dimensions: the corrected antidiffusive flux approach, Math. Comp. 57 (1991), 169-210. MR 91m:65229

9. R.J. DiPerna, Measure-valued solutions to conservations laws, Arch. Rational Mech. Anal. 88 (1985), 223-270. MR 86g:35121

10. B. Engquist and S. Osher, One-sided difference approximations for nonlinear conservation laws, Math. Comp. 36 (1981), 321-351. MR 82c:65056 
11. T. Geveci, The significance of the stability of difference schemes in different $l^{p}$-spaces, SIAM Rev. 24 (1982), 413-426. MR 84b:65089

12. A. Harten, J. M. Hyman and P. Lax, On finite difference approximations and entropy conditions for shocks, Comm. Pure Appl. Math. 29 (1976), 297-322. MR 54:1640

13. S.N. Kröner and M. Rokyta, Convergence of upwind finite volume methods for scalar conservation laws in two dimensions, SIAM J. Numer. Anal. 31 (1994), 324-343. MR 95e:65085

14. S.N. Kružkov, First order quasilinear equations in several independent variables, Math. USSR Sbornik 10 (1970), 217-243. MR 42:2159

15. N.N. Kuznetsov, Accuracy of some approximate methods for computing the weak solutions of a first-order quasi-linear equation, USSR Comput. Math. and Math. Phys. 16 (1976), 105-119. MR 58:3510

16. B. J. Lucier, A stable adaptive scheme for hyperbolic conservation laws, SIAM J. Numer. Anal. 22 (1985), 180-203. MR 86d:65123

17. _ Error bounds for the methods of Glimm, Godunov and LeVeque, SIAM J. Numer. Anal. 22 (1985), 1074-1081. MR 88a:65104

18. 47 (1986), 19-36. MR 87j:65110

19. __ A moving mesh numerical method for hyperbolic conservation laws, Math. Comp. 46 (1986), 59-69. MR 87m:65141

20. S. Osher, Riemann solvers, the entropy condition, and difference approximations, SIAM J. Numer. Anal. 21 (1984), 217-235. MR 86d:65119

21. B. Perthame and R. Sanders, The Neumann problem for nonlinear second-order singular perturbation problems, SIAM J. Math. Anal. 19 (1988), 295-311. MR 89d:35012

22. R. Sanders, On convergence of monotone finite difference schemes with variable spatial differencing, Math. Comp. 40 (1983), 91-106. MR 84a:65075

23. A. Szepessy, Convergence of a shock-capturing streamline diffusion finite element method for scalar conservation laws in two space dimensions, Math. Comp. 53 (1989), 527-545. MR 90h:65156

24. Convergence of a streamline diffusion finite element method for a conservation law with boundary conditions, (RAIRO) Model. Math. Anal. Numer. 25 (1991), 749-783. MR 92g:65115

25. E. Tadmor and T. Tassa, On the piecewise smoothness of entropy solutions to scalar conservation laws, Comm. Partial Differential Equations 18 (1993), 1631-1652. MR 94g:35144

26. J.-P. Vila, Convergence and error estimates in finite volume schemes for general multidimensional scalar conservation laws, Model. Math. Anal. Numer. 28 (1994), 267-295. CMP 94:12

27. A. I. Vol'pert and S. I. Hudjaev, Cauchy's problem for degenerate second order quasilinear parabolic equations, Math. USSR Sbornik 7 (1969), 365-387. MR 41:8828

School of Mathematics, University of Minnesota, 127 Vincent Hall, Minneapolis, Minnesota 55455

E-mail address: cockburn@math.umn.edu

Department of Mathematics, North Carolina State University, Box 8205, Raleigh, North CARolina 27695-8205

E-mail address: gremaud@dali.math.ncsu.edu 